\title{
STAR FORMATION PROPERTIES IN BARRED GALAXIES. III. STATISTICAL STUDY OF BAR-DRIVEN SECULAR EVOLUTION USING A SAMPLE OF NEARBY BARRED SPIRALS
}

\author{
Zhi-Min Zhou ${ }^{1}$, Chen $\mathrm{CAO}^{2,3,4}$, AND Hong Wu ${ }^{1}$ \\ ${ }^{1}$ Key Laboratory of Optical Astronomy, National Astronomical Observatories, Chinese Academy of Sciences, \\ Beijing 100012, China; zmzhou@bao.ac.cn, hwu@bao.ac.cn \\ ${ }_{3}^{2}$ School of Space Science and Physics, Shandong University at Weihai, Weihai, Shandong 264209, China; caochen@sdu.edu.cn \\ ${ }^{3}$ Shandong Provincial Key Laboratory of Optical Astronomy \& Solar-Terrestrial Environment, Weihai, Shandong 264209, China \\ ${ }^{4}$ Visiting Scholar, Infrared Processing and Analysis Center (IPAC), California Institute of Technology, Pasadena, CA 91125, USA \\ Received 2014 March 18; accepted 2014 September 9; published 2014 November 25
}

\begin{abstract}
Stellar bars are important internal drivers of secular evolution in disk galaxies. Using a sample of nearby spiral galaxies with weak and strong bars, we explore the relationships between the star formation feature and stellar bars in galaxies. We find that galaxies with weak bars tend coincide with low concentrical star formation activity, while those with strong bars show a large scatter in the distribution of star formation activity. We find enhanced star formation activity in bulges toward stronger bars, although not predominantly, consistent with previous studies. Our results suggest that different stages of the secular process and many other factors may contribute to the complexity of the secular evolution. In addition, barred galaxies with intense star formation in bars tend to have active star formation in their bulges and disks, and bulges have higher star formation densities than bars and disks, indicating the evolutionary effects of bars. We then derived a possible criterion to quantify the different stages of the bar-driven physical process, while future work is needed because of the uncertainties.
\end{abstract}

Key words: galaxies: evolution - galaxies: general - galaxies: photometry - galaxies: spiral - galaxies: structure

\section{INTRODUCTION}

Stellar bars are ubiquitous in disk galaxies. Observed bar fractions range from one- to two-thirds in the local universe depending on the method used to identify bars and the sample and wave-bands observed (Eskridge et al. 2000; MenéndezDelmestre et al. 2007). Bars are also found in galaxies up to $z$ $\sim 1$ (Sheth et al. 2008; Melvin et al. 2014). Bars are one of the major internal drivers of secular evolution in galaxies (see Kormendy \& Kennicutt 2004 for a review). The nonaxisymmetric gravitational potentials of bars can redistribute the angular momentum of gas and stars in galactic disks, induce large-scale streaming motions (Regan et al. 1995, 1999; Sakamoto et al. 1999b; Sheth et al. 2005), and result in an increase in gas mass and enhancement of star formation activity in the galactic central regions (Sérsic \& Pastoriza 1965; Hawarden et al. 1986; Ho et al. 1997).

Many studies, both theoretical and observational, have been undertaken to explore the process of bar-driven secular evolution. Numerical simulations have established that the gravitational torque of large-scale bars can make gas lose angular momentum and allow it to move inward toward the galactic center (Athanassoula 1992; Sellwood \& Wilkinson 1993; Piner et al. 1995; Athanassoula 2003). Observations indicate that barred spirals have higher molecular gas concentrations in their central kiloparsecs than unbarred systems (Sakamoto et al. 1999b; Sheth et al. 2005). Younger stellar populations and higher star formation rates (SFRs) are also found in the bulges of barred galaxies compared with unbarred ones (e.g., Sérsic \& Pastoriza 1965; Huang et al. 1996; Alonso-Herrero \& Knapen 2001; Kormendy \& Fisher 2005; Fisher 2006; Wang 2008; Coelho \& Gadotti 2011; Wang et al. 2012). Kormendy \& Kennicutt (2004) showed correlations between the surface densities of SFR and gas and compared these correlations in the circumnuclear star-forming rings in barred galaxies with those in the disks of spiral galaxies. They found that both SFR densities and gas densities in circumnuclear rings are higher than those in the outer disks.

However, some observations found conflicting results as to how a bar affects the evolution of disk galaxies. Ho et al. (1997) showed that the bars in late-type spirals have no noticeable impact on the likelihood of a galaxy for hosting nuclear star formation. Furthermore, there are some barred galaxies with no molecular gas detected in their nuclear regions where it may have been consumed by star formation (Sheth et al. 2005). Using emission-line diagnostics to identify central star formation activity in galaxies, Oh et al. (2012) found that bar effects on central star formation seem higher in redder galaxies and are more pronounced with increasing bar length, but the effects on central star formation are not visible in blue galaxies. Similarly, Wang et al. (2012) found that only strong bars result in enhanced central star formation in galaxies, but not weak bars. Ellison et al. (2011) found enhanced fiber SFR and metallicities just exist in massive barred galaxies, and no significant correlation between SFR enhancement and bar length or ellipticity. Based on the Galaxy Zoo 2 data set, Cheung et al. (2013) studied the behavior of bars in disk galaxies and found that the trends of bar likelihood and bar length with bulge prominence are bimodal with specific star formation rates (SSFRs).

In previous studies, comparisons were often made between barred and unbarred galaxies, whereas the comparisons between the variation in stellar bar properties and their associated star formation are crucial to the understanding of the bar-driven secular process in galaxies. Thus, a large sample and multi-wavelength data are required. In our previous work, three barred galaxies in different evolutionary stages were analyzed based on the data in different wavelength bands: NGC 7479 in Zhou et al. (2011, hereafter Paper I) and NGC 2903 and NGC 7080 in Zhou et al. (2012, hereafter Paper II). In this 
paper, we use multi-wavelength data from the optical to the infrared (IR) to analyze a relatively large sample of nearby barred galaxies (with both weak and strong bars, including early to late Hubble types). We aim to statistically study the correlation between star formation activity and bar properties, especially its strength.

This paper is organized as follows. In Section 2, we describe the selection of the sample and give a brief introduction of the data. In Section 3, we present the method used for image decomposition and derive the structural characterization of our galaxies. We explore the correlations between the star formation and bar properties in Section 4. Then we discuss our results and explore the possible evolutionary episodes of bar-driven secular evolution in Section 5. Finally, we finish with our summary and conclusions in Section 6.

\section{SAMPLE AND DATA}

\subsection{Sample Selection}

Our sample is mainly selected from three Spitzer local programs: the Spitzer Infrared Nearby Galaxies Survey (SINGS; Kennicutt et al. 2003), the Local Volume Legacy (LVL; Lee 2008; Dale et al. 2009), and the Infrared Hubble Atlas (Pahre et al. 2004b). From all nearby galaxies in these programs, we selecte galaxies with bars, i.e., with Hubble type of SB and SAB. In addition, we apply an inclination cut to remove apparently edge-on galaxies (inclination $>65^{\circ}$ or ellipticity < 0.58), as well as removing objects that are obviously merging. This produces the final sample size of 50 galaxies.

Table 1 shows the general properties of the 50 galaxies in our sample (see Figure 1). The distances given in Table 1 were luminosity distances from the NASA Extragalactic Database (NED). ${ }^{5}$ The distance of the studied sample has a mean value of $20.5 \mathrm{Mpc}$ and a maximum value of $63.6 \mathrm{Mpc}$. The standard isophotal galaxy diameters $\left(D_{25}\right)$ are $\sim 3^{\prime}$, with all sizes larger than $1^{\prime}$. Galaxies in the sample include a wide variety of Hubble types. There are $27 \mathrm{SB}$ galaxies and $23 \mathrm{SAB}$ galaxies ranging from $\mathrm{Sa}$ to $\mathrm{Sm}$, corresponding to the numerical morphological index $\mathrm{T}=1-9$. Figure 2 shows the distribution of morphology for all sample galaxies. In the following analysis we divided galaxies into early-type $(T \leqslant 4)$ and latetype $(T \geqslant 5)$ spirals. Thus, there are 30 early-type spirals and 20 late-type spirals in our sample. In the right panel of Figure 2, we also plotted the distance and magnitude distribution of our sample.

\subsection{Data Acquisition and Reduction}

In order to investigate the properties of barred galaxies, we archived IR images from the Spitzer Space Telescope (Werner et al. 2004) data archive. In optical wavelength, the narrowband $\mathrm{H} \alpha$ images from ground-based telescopes are collected and used to analyze the star formation activity in galaxies. In the following section, we provide the multi-wavelength data observation/acquisition and a brief description of data reduction. A more detailed description of data acquisition and reduction can be found in Papers I and II.

\footnotetext{
http://nedwww.ipac.caltech.edu/
}

\subsubsection{Optical Data}

In our sample, 17 galaxies were observed with the $2.16 \mathrm{~m}$ telescope at Xinglong Observatory of the National Astronomical Observatories of the Chinese Academy of Sciences ${ }^{6}$ during 2009-2010. The instrument we used was the BAO Faint Object Spectrograph and Camera (BFOSC). It had a Lick $2048 \times 2048$ CCD detector with a plate scale of $0 .^{\prime \prime} 305$ pixel $^{-1}$ and a field of view of $\sim 10^{\prime} \times 10^{\prime}$. The galaxies were imaged with the broadband $R$ filter and narrowband filters centered on the appropriate redshift $\mathrm{H} \alpha+[\mathrm{N}$ II $] \lambda \lambda 6563,6583$ emission lines; the former images were used to subtract the stellar continuum from the $\mathrm{H} \alpha+[\mathrm{N}$ II] images. The exposure time was typically $600 \mathrm{~s}$ for $R$-band and $3000-3600 \mathrm{~s}$ for $\mathrm{H} \alpha$-band. Detailed information about the observations is listed in Table 2 .

The IRAF $^{7}$ package was used for data reduction. The reduction pipeline includes checking images, adding keywords to fits headers, subtracting overscan and bias, correcting bad pixels and flat-fielding, along with removing cosmic-rays. The flux calibration of broadband images was made using the standard stars selected from the Landolt fields (Landolt 1992) and observed with the corresponding filter on the same night. After sky subtraction, the scaled $R$-band images were subtracted from the aligned narrowband images to obtain continuum-free $\mathrm{H} \alpha+\left[\mathrm{N}_{\mathrm{NI}}\right]$ line images, and the scale factors were calculated using photometric count ratios of several $(\sim 5)$ unsaturated field stars in both filter images. The narrowband images were flux calibrated based on the scale factors of $R$ band images and effective transmissions of narrowband and $R$ band filters. Comparing the calibrated $\mathrm{H} \alpha$ and $R$-band images indicates that there is $\sim 6 \%$ of $\mathrm{H} \alpha$ emission lost in the process of removing stellar continuum, while the contribution from $[\mathrm{N}$ II] to the total $\mathrm{H} \alpha$ flux is typically of the order of $10 \%$ or less (Kennicutt et al. 2008). Thus, the final images have spatial resolutions of $\sim 2^{\prime \prime}$ along with a flux uncertainty of $\sim 10 \%$.

In addition to our observations, optical data in $R$ broadband and $\mathrm{H} \alpha$ narrowband filters were gathered in other previous studies and are listed in Table 3. Images of 16 galaxies from the SINGS sample were obtained from the ancillary data of the legacy project; these galaxies were observed by the Kitt Peak National Observatory (KPNO) $2.1 \mathrm{~m}$ telescope and the Cerro Tololo Inter-American Observatory (CTIO) $1.5 \mathrm{~m}$ telescope. Images of eight galaxies from the LVL sample were obtained from the ancillary data of the LVL project as part of the precursor $11 \mathrm{Mpc} \mathrm{H} \alpha$ Survey (Kennicutt et al. 2008). The optical data of other sample galaxies were derived from NED. The observations, data processing, and flux calibration of the optical archival images nicely matched the counterparts of our own data.

\subsubsection{IR Data}

To investigate the infrared properties of our sample, we retrieved the available archived Spitzer near-/mid-IR data from the SINGS and LVL Program data sets, including the Infrared Array Camera (IRAC; Fazio et al. 2004) imaging data at 3.6, 4.5, 5.8, and $8.0 \mu \mathrm{m}$ bands, as well as the Multiband Imaging Photometer for Spitzer (MIPS; Rieke et al. 2004) imaging data

\footnotetext{
6 http://www.xinglong-naoc.org/English/216.html

7 IRAF is the Image Reduction and Analysis Facility written and supported by the IRAF programming group at the National Optical Astronomy Observatories (NOAO) in Tucson, AZ which is operated by AURA, Inc. under cooperative agreement with the National Science Foundation
} 
Table 1

Basic Parameters of the Whole Sample

\begin{tabular}{|c|c|c|c|c|c|c|c|c|}
\hline Galaxy (name) & R.A. (J2000.0) & Decl. (J2000.0) & Morphology (RC3) & $\mathrm{R}_{25}{ }^{\mathrm{a}}$ (arcseconds) & $e^{\mathrm{b}}(\operatorname{deg})$ & P.A. ${ }^{\mathrm{c}}(\mathrm{Mpc})$ & $D^{d}$ & Source $^{\mathrm{e}}$ (ref) \\
\hline NGC 0023 & 00:09:53.40 & $+25: 55: 26$ & $\mathrm{SBa}$ & 62.68 & 0.35 & -30 & 58.60 & 4 \\
\hline NGC 0266 & $00: 49: 47.80$ & $+32: 16: 40$ & $\mathrm{SBa}$ & 88.54 & 0.02 & 0 & 60.40 & 3 \\
\hline NGC 0337 & $00: 59: 50.10$ & $-07: 34: 41$ & SBd & 86.52 & 0.37 & -50 & 18.30 & 1 \\
\hline NGC 1097 & $02: 46: 19.00$ & $-30: 16: 30$ & $\mathrm{SBb}$ & 279.98 & 0.32 & -60 & 15.20 & 1 \\
\hline NGC 1512 & 04:03:54.30 & $-43: 20: 56$ & SBab & 267.38 & 0.37 & 90 & 11.70 & 1 \\
\hline NGC 1566 & $04: 20: 00.40$ & $-54: 56: 16$ & $\mathrm{SABbc}$ & 249.53 & 0.21 & 60 & 20.60 & 1 \\
\hline NGC 2403 & $07: 36: 51.40$ & $+65: 36: 09$ & SABcd & 656.33 & 0.44 & -53 & 2.47 & 1 \\
\hline NGC 2903 & 09:32:10.10 & $+21: 30: 04$ & SBd & 377.68 & 0.52 & 17 & 11.70 & 2 \\
\hline NGC 2964 & $09: 42: 54.20$ & $+31: 50: 50$ & $\mathrm{SABbc}$ & 86.52 & 0.45 & -83 & 21.90 & 3 \\
\hline NGC 3049 & $09: 54: 49.50$ & $+09: 16: 16$ & SBab & 65.63 & 0.34 & 22 & 24.70 & 1 \\
\hline NGC 3184 & $10: 18: 17.00$ & $+41: 25: 28$ & SABcd & 222.39 & 0.07 & -45 & 11.40 & 1 \\
\hline NGC 3198 & $10: 19: 54.90$ & $+45: 32: 59$ & $\mathrm{SBc}$ & 255.34 & 0.61 & 37 & 12.10 & 1 \\
\hline NGC 3274 & $10: 32: 17.10$ & $+27: 40: 07$ & SABd & 64.14 & 0.52 & -80 & 11.50 & 2 \\
\hline NGC 3319 & $10: 39: 09.40$ & $+41: 41: 12$ & SBcd & 184.98 & 0.45 & 37 & 13.50 & 3 \\
\hline NGC 3344 & $10: 43: 31.10$ & $+24: 55: 20$ & $\mathrm{SABbc}$ & 212.38 & 0.09 & 0 & 12.30 & 2 \\
\hline NGC 3351 & $10: 43: 57.70$ & $+11: 42: 14$ & $\mathrm{SBb}$ & 222.39 & 0.32 & 13 & 15.50 & 1 \\
\hline NGC 3368 & $10: 46: 45.70$ & $+11: 49: 12$ & SABab & 227.57 & 0.31 & 5 & 17.10 & 2 \\
\hline NGC 3486 & 11:00:23.90 & $+28: 58: 29$ & $\mathrm{SABc}$ & 212.38 & 0.26 & 80 & 13.50 & 2 \\
\hline NGC 3521 & $11: 05: 48.60$ & $-00: 02: 09$ & SABbc & 328.94 & 0.53 & -17 & 16.10 & 1 \\
\hline NGC 3627 & $11: 20: 15.00$ & $+12: 59: 30$ & $\mathrm{SABb}$ & 273.6 & 0.54 & -7 & 14.80 & 1 \\
\hline NGC 4020 & $11: 58: 56.60$ & $+30: 24: 44$ & SBd & 62.68 & 0.55 & 15 & 14.40 & 2 \\
\hline NGC 4051 & $12: 03: 09.60$ & $+44: 31: 53$ & SABbc & 157.44 & 0.26 & -45 & 12.70 & 4 \\
\hline NGC 4136 & 12:09:17.70 & $+29: 55: 39$ & $\mathrm{SABc}$ & 119.43 & 0.07 & 0 & 12.30 & 3 \\
\hline NGC 4288 & $12: 20: 38.10$ & $+46: 17: 33$ & SBdm & 64.14 & 0.24 & -50 & 10.00 & 2 \\
\hline NGC 4303 & $12: 21: 54.90$ & $+04: 28: 25$ & SABbc & 193.7 & 0.11 & 0 & 26.40 & 3 \\
\hline NGC 4314 & $12: 22: 32.00$ & $+29: 53: 43$ & $\mathrm{SBa}$ & 125.06 & 0.11 & -34 & 17.10 & 3 \\
\hline NGC 4394 & $12: 25: 55.60$ & $+18: 12: 50$ & $\mathrm{SBb}$ & 108.92 & 0.11 & -40 & 17.00 & 4 \\
\hline NGC 4491 & $12: 30: 57.10$ & $+11: 29: 01$ & $\mathrm{SBa}$ & 50.95 & 0.50 & -32 & 11.40 & 4 \\
\hline NGC 4535 & $12: 34: 20.30$ & $+08: 11: 52$ & $\mathrm{SABc}$ & 212.38 & 0.29 & 0 & 31.70 & 4 \\
\hline NGC 4548 & $12: 35: 26.40$ & $+14: 29: 47$ & $\mathrm{SBb}$ & 161.11 & 0.21 & -30 & 11.10 & 4 \\
\hline NGC 4569 & $12: 36: 49.80$ & $+13: 09: 46$ & $\mathrm{SABab}$ & 286.5 & 0.54 & 23 & 17.00 & 1 \\
\hline NGC 4579 & $12: 37: 43.50$ & $+11: 49: 05$ & $\mathrm{SABb}$ & 176.65 & 0.21 & -85 & 25.40 & 1 \\
\hline NGC 4580 & $12: 37: 48.40$ & $+05: 22: 07$ & SABa & 62.68 & 0.22 & -15 & 18.90 & 4 \\
\hline NGC 4647 & $12: 43: 32.30$ & $+11: 34: 55$ & $\mathrm{SABc}$ & 86.52 & 0.21 & -55 & 23.90 & 3 \\
\hline NGC 4654 & $12: 43: 56.60$ & $+13: 07: 36$ & SABcd & 146.93 & 0.43 & -52 & 18.80 & 3 \\
\hline NGC 4725 & $12: 50: 26.60$ & $+25: 30: 03$ & SABab & 321.46 & 0.29 & 35 & 20.50 & 1 \\
\hline NGC 5248 & $13: 56: 16.70$ & $+47: 14: 08$ & $\mathrm{SBa}$ & 184.98 & 0.28 & -50 & 19.80 & 3 \\
\hline NGC 5377 & $13: 37: 32.10$ & $+08: 53: 06$ & $\mathrm{SBbc}$ & 111.46 & 0.44 & 45 & 26.80 & 3 \\
\hline NGC 5457 & $14: 03: 12.60$ & $+54: 20: 57$ & SABcd & 865.21 & 0.07 & 90 & 4.97 & 2 \\
\hline NGC 5713 & $14: 40: 11.50$ & $-00: 17: 21$ & SABbc & 82.63 & 0.11 & 10 & 29.30 & 1 \\
\hline NGC 5832 & $14: 57: 45.70$ & $+71: 40: 56$ & $\mathrm{SBb}$ & 111.46 & 0.41 & 45 & 6.43 & 2 \\
\hline NGC 6701 & $18: 43: 12.40$ & $+60: 39: 12$ & $\mathrm{SBa}$ & 46.46 & 0.15 & -67 & 53.40 & 4 \\
\hline NGC 6946 & $20: 34: 52.30$ & $+60: 09: 14$ & SABcd & 344.45 & 0.15 & -10 & 6.90 & 1 \\
\hline NGC 7080 & 21:30:01.90 & $+26: 43: 04$ & $\mathrm{SBb}$ & 54.59 & 0.05 & 90 & 62.70 & 3 \\
\hline NGC 7479 & $23: 04: 56.60$ & $+12: 19: 22$ & $\mathrm{SBc}$ & 122.21 & 0.24 & 9 & 27.70 & 3 \\
\hline NGC 7552 & $23: 16: 10.80$ & $-42: 35: 05$ & SBab & 101.65 & 0.21 & 90 & 18.80 & 1 \\
\hline NGC 7591 & $23: 18: 16.30$ & $+06: 35: 09$ & $\mathrm{SBbc}$ & 58.5 & 0.57 & -35 & 63.60 & 4 \\
\hline NGC 7741 & $23: 43: 54.40$ & $+26: 04: 32$ & SBcd & 130.95 & 0.32 & -10 & 5.53 & 3 \\
\hline NGC 7798 & $23: 59: 25.50$ & $+20: 44: 59$ & $\mathrm{SBc}$ & 41.41 & 0.09 & 90 & 28.30 & 3 \\
\hline UGC 01249 & $01: 47: 30.60$ & $+27: 19: 52$ & $\mathrm{SBm}$ & 207.55 & 0.55 & -30 & 0.96 & 2 \\
\hline
\end{tabular}

${ }^{a}$ Optical radii from RC3.

${ }^{\mathrm{b}}$ Ellipticity from RC3.

${ }^{\mathrm{c}}$ Position angle from RC3.

${ }^{d}$ The luminosity distance from NED

${ }^{\mathrm{e}}$ References from which objects were selected: (1) Kennicutt et al. (2003), (2) Lee (2008), (3) Pahre et al. (2004b), (4) Sanders et al. (2003).

at $24 \mu \mathrm{m}$ band. The IRAC images at each channel had a pixel scale of 0.75 , and spatial resolutions of $\sim 2 . \prime 0$, which are similar to those of optical $\mathrm{H} \alpha$ images. The $24 \mu \mathrm{m}$ MIPS images had a pixel scale of 1."5, with an FWHM of $\sim 6 . " 0$.

For those galaxies that are not from the SINGS or LVL surveys, we obtained the basic calibrated data (BCD) generated from the Spitzer data reduction pipeline and then mosaicked them with the MOsaicker and Point source Extractor (MOPEX) software. The final images were produced with the same plate scales and spatial resolutions similar to the archived infrared data.

In IRAC $8.0 \mu \mathrm{m}$ band images, the flux is mainly from the polycyclic aromatic hydrocarbon (PAH) emission and dustcontinuum emission (Pahre et al. 2004a, 2004b), but there is 


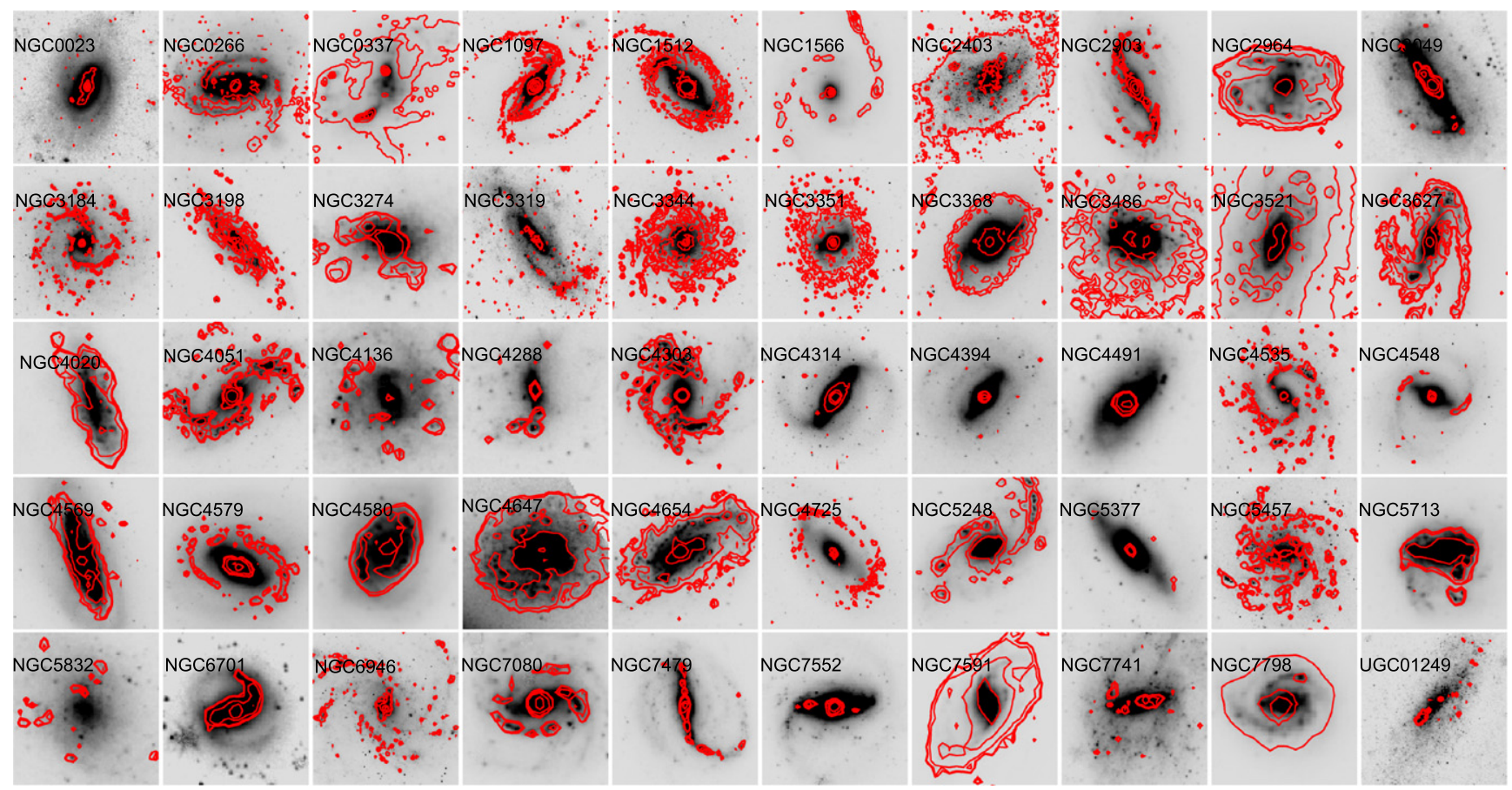

Figure 1. Mass images of the galaxies in our sample. Overplotted are the SFR imaging contours, which are in arbitrary units, optimized to show and identify the structures of the star formation complexes. The galaxy names are specified in the panels and north is up and east is to the left.
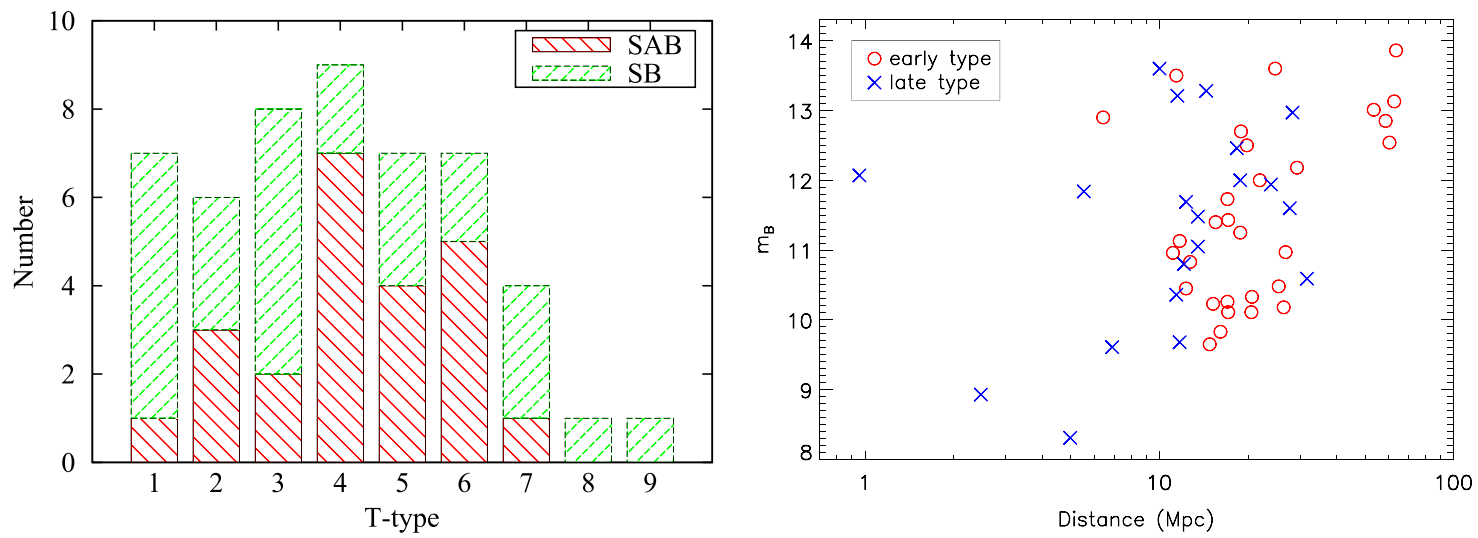

Figure 2. Morphological distribution (left) and magnitude-distance distribution (right) of our sample. In the left panel, the morphological parameter $\mathrm{T}=1-9$ corresponds to the Hubble types Sa-Sm, and the sample is divided into SB and SAB galaxies. In the right panel, the distance used is luminosity distance from NED, and the sample is divided into early $(\mathrm{T}=1-4)$ and late $(\mathrm{T}=5-9)$ types with red circles for early-type galaxies and blue crosses for late-type ones.

also a contribution of stellar continuum in this band. To remove the stellar contribution, the scaled IRAC $3.6 \mu \mathrm{m}$ band images were used as the stellar continuum with a scale factor of 0.232 (Helou et al. 2004) and were subtracted from $8.0 \mu \mathrm{m}$ images. Hereafter we referred to the continuum-free $8 \mu \mathrm{m}$ dust emission as to $8 \mu \mathrm{m}$ (dust).

\section{IMAGE DECOMPOSITION AND BAR CHARACTERIZATION}

We derive the structural parameters of bars, i.e., the length, ellipticity, and strength, with structural decomposition of barred galaxies. The IRAC $3.6 \mu \mathrm{m}$ images, wherein the light is mainly coming from $\mathrm{M}$ and $\mathrm{K}$ giants (Sheth et al. 2010), nicely trace the stellar mass distribution in nearby galaxies although there is contamination from the $3.3 \mu \mathrm{m} \mathrm{PAH}$, hot dust, and intermediate-age stars (Meidt et al. 2012). Therefore, we used the image-fitting code BUDDA v2.2 (de Souza et al. 2004; Gadotti 2008) to perform a two-dimensional (2D) decomposition of $3.6 \mu \mathrm{m}$ images in bar/bulge/disk stellar components. The present version of BUDDA allows us to decompose a galaxy into an exponential disk, a bulge with a Sérsic profile, and a bar (e.g., Kim et al. 2014). In the decomposition results, we obtain the galaxy structural parameters, such as the size, ellipticity, and position angle (P.A.) of each structure, which were also used to define the regions of the bulge, bar, and disk in one galaxy.

The structural parameters we obtain may be affected by inclination effects. To derive the intrinsic physical parameters, we make the corrections for the apparent values following the method of Martin (1995). In Martin (1995), the galaxy inclination $i$ was defined using the axial ratio of the isophote $25 \mathrm{mag} \mathrm{arcsec}^{-2}$ in the $B$-band obtained from the Third 
Table 2

XingLong $2.16 \mathrm{~m}$ Telescope Observations

\begin{tabular}{|c|c|c|c|c|c|}
\hline \multirow[b]{2}{*}{$\begin{array}{l}\text { Name } \\
(1)\end{array}$} & \multirow[b]{2}{*}{$\begin{array}{c}\text { Obs. Date } \\
\text { (2) }\end{array}$} & \multicolumn{2}{|c|}{$\begin{array}{l}\text { Exposure } \\
\text { Time } \\
\text { (Section) }\end{array}$} & \multirow{2}{*}{$\begin{array}{c}\mathrm{H} \alpha \text { Filter } \\
\lambda / \Delta \lambda \\
(5)\end{array}$} & \multirow{2}{*}{$\begin{array}{c}\text { Seeing } \\
\text { (arcseconds) } \\
(6)\end{array}$} \\
\hline & & $\begin{array}{l}\mathrm{H} \alpha \\
(3)\end{array}$ & $\begin{array}{l}\mathrm{R} \\
(4)\end{array}$ & & \\
\hline NGC 0023 & 2006-09-22 & 2400 & 400 & $6660 / 70$ & 2.0 \\
\hline NGC 0266 & 2008-01-10 & 3600 & 600 & $6660 / 70$ & 2.7 \\
\hline NGC 2903 & $2009-12-22$ & 3600 & 600 & $6562 / 70$ & 1.7 \\
\hline NGC 2964 & 2009-01-18 & 2400 & 600 & $6610 / 70$ & 2.0 \\
\hline NGC 3319 & 2007-03-17 & 3600 & 600 & $6562 / 70$ & 1.9 \\
\hline NGC 4303 & 2007-02-24 & 1800 & 600 & $6610 / 70$ & 2.6 \\
\hline NGC 4491 & 2009-04-21 & 3600 & 600 & $6562 / 70$ & 2.3 \\
\hline NGC 4580 & 2009-04-21 & 3600 & 600 & $6610 / 70$ & 2.3 \\
\hline NGC 4647 & 2009-01-18 & 1800 & 600 & $6610 / 70$ & 2.0 \\
\hline NGC 4654 & $2008-02-14$ & 3600 & 600 & $6562 / 70$ & 2.1 \\
\hline NGC 5377 & $2008-02-12$ & 3000 & 600 & $6610 / 70$ & 3.0 \\
\hline NGC 5383 & 2009-04-21 & 1800 & 600 & $6610 / 70$ & 1.8 \\
\hline NGC 6701 & $2006-07-22$ & 2400 & 400 & $6660 / 70$ & 2.6 \\
\hline NGC 7080 & $2006-09-23$ & 2400 & 400 & $6660 / 70$ & 1.8 \\
\hline NGC 7479 & 2009-09-12 & 3000 & 600 & $6610 / 70$ & 1.8 \\
\hline NGC 7591 & 2006-09-22 & 3600 & 400 & $6660 / 70$ & 2.4 \\
\hline NGC 7798 & $2006-08-21$ & 2400 & 600 & $6610 / 70$ & 2.7 \\
\hline
\end{tabular}

Notes. Col. (1): name of the galaxy. Col. (2): the date of the observation. Cols. (3) and (4): exposure time in each filter in units of seconds. Col. (5): filter used for the $\mathrm{H} \alpha$ line, where $\lambda \Delta \lambda$ is the central wavelength and filter width in $\AA$. Col. (6): seeing in arcseconds as measured on the reduced images.

Reference Catalog of Bright Galaxies (RC3; de Vaucouleurs et al. 1991) and the assumption that the galaxy is intrinsically axisymmetric at large radii. Given that the bars are thin 2D structures, the intrinsic axial ratio $(\mathrm{b} / \mathrm{a})_{\mathrm{bar}}$ and length $\mathrm{r}_{\mathrm{bar}}$ (the semimajor axis) of bars were calculated with the galaxy inclination and angles between the axes and line of nodes. Figure 3 compares the bar properties before and after correction. There is a large correction on the axial ratio of bars, but there is excellent consistency between the lengths of bars.

Figure 4 shows the distribution of the intrinsic bar lengths of the sample, both absolute and normalized. The latter is relative to the galaxy size $R_{25}$, which is measured by the semimajor axis of the isophote from RC3 at a brightness of $25 \mathrm{mag} \operatorname{arcsec}^{-2}$ in the $B$-band. The mean length of stellar bars is $5.2 \mathrm{kpc}$ and $0.37 \mathrm{R}_{25}$ in early types and $2.2 \mathrm{kpc}$ and 0.23 $\mathrm{R}_{25}$ in late types, consistent with values in earlier studies (Erwin 2005; Menéndez-Delmestre et al. 2007).

Bar strength represents the ability to drive gas into the galactic center and is usually estimated using the structural parameters of the bar such as ellipticity and length of the bar (e.g., Jogee et al. 2004; Laurikainen et al. 2007). A more direct parameter is the gravitational torque $\mathrm{Q}_{g}$, which is the maximum relative tangential force in the bar region, normalized to the underlying mean axisymmetric force field (Buta \& Block 2001). Here, another bar strength parameter is used and is defined as follows Abraham \& Merrifield (2000):

$$
f_{\mathrm{bar}}=\frac{2}{\pi}\left[\arctan (b / a)_{\mathrm{bar}}^{-1 / 2}-\arctan (b / a)_{\mathrm{bar}}^{+1 / 2}\right],
$$

where $(b / a)_{b a r}$ is the intrinsic axial ratio of the bar. The ellipticity-based parameter $f_{\text {bar }}$ has been used in many previous studies (e.g., Whyte et al. 2002; Aguerri et al. 2009) and has proved to be well correlated with $\mathrm{Q}_{g}$ (Buta et al. 2004; Laurikainen et al. 2007). The right panel of Figure 4 shows the distribution of the bar strength $f_{\text {bar }}$. Bars in early-type galaxies are clearly stronger than those in late-type galaxies. Besides the parameter $f_{\text {bar }}$ and bar torque, the normalized bar size $\eta_{\text {bar }} / r_{\text {gal }}$ and the combination of bar ellipticity and boxiness $\epsilon \times c$ were also used in Gadotti (2011) to estimate bar strength. We compare the two parameters with $f_{\text {bar }}$ in Figure 5. This figure shows that $f_{\text {bar }}$ is consistent with the other two parameters, especially $\epsilon \times c$, so it is used as the estimation of bar strength in the following analysis. Their values are also listed in Table 4.

\section{RELATION BETWEEN GALACTIC STAR FORMATION PROPERTIES AND BARS}

Star formation activity is an important feature in our galaxies. In our data of the sample, the $\mathrm{H} \alpha$ emission from ground-based observations and the $8 \mu \mathrm{m}$ (dust) and $24 \mu \mathrm{m}$ emission from Spitzer observations can be used as star formation tracers (Kennicutt 1998; Wu et al. 2005; Calzetti et al. 2007; Zhu et al. 2008; Kennicutt et al. 2009; Hao et al. 2011). To derive the extinction-corrected SFR distribution, we used a combination of $\mathrm{H} \alpha$ and $8 \mu \mathrm{m}$ (dust) emissions, the former measuring the ionization rate and the latter tracing the dust-obscured component of the star formation. The calibration for the composite indicators follows the form in Zhu et al. (2008):

$$
\begin{aligned}
& \mathrm{SFR}_{H \alpha+8 \mu \mathrm{m}}\left(M_{\odot} \mathrm{yr}^{-1}\right) \\
& \quad=7.9 \times 10^{-42}\left[L(H \alpha)_{\mathrm{obs}}+0.010 L(8)\right]\left(\mathrm{erg} \mathrm{s}^{-1}\right),
\end{aligned}
$$

which adopts the Salpeter (1955) initial mass function (IMF) with a slope of 2.35 for stellar masses in the range $0.1-100 M_{\odot}$.

In addition to the star formation activity, we also paid attention to the stellar mass of galaxies to derive the stellar properties. Here, the IRAC $3.6 \mu \mathrm{m}$ emission is used as a proxy of the stellar mass since its light is dominated by the photospheric emission from low-mass stars as mentioned above (Smith et al. 2007; Hancock et al. 2007; Wu et al. 2007; Li et al. 2007). The calibration is from Zhu et al. (2010; their Equation (2)):

$$
\begin{aligned}
\log _{10} M\left(M_{\odot}\right)= & (-0.79 \pm 0.03)+(1.19 \pm 0.01) \\
& \times \log _{10} \nu L_{\nu}[3.6 \mu \mathrm{m}]\left(L_{\odot}\right)
\end{aligned}
$$

which has proved to be a good stellar mass tracer compared with $K_{s}$-band luminosity. Using these Equations, we explored the correlation between the star formation activity and stellar bars in our galaxies.

\subsection{Star Formation and Stellar Mass Distribution}

In order to describe the global properties of the galaxies, we first obtained the mass images and SFR images by combining the relevant images based on Equations (3) and (2), respectively. All images are listed in Figure 1, along with the distributions of stellar mass and star formation shown. Then we calculated the rotational asymmetry and concentration index of the mass and SFR images to quantify their distributions. The asymmetry describes how symmetric a galaxy system is and the concentration parameter measures how compact the light distribution is. Both indices are used to describe galaxy 
Table 3

Details of the Archival $\mathrm{H} \alpha$ Images Obtained For Our Sample Galaxies

\begin{tabular}{|c|c|c|c|c|c|c|}
\hline \multirow{3}{*}{$\begin{array}{l}\text { Name } \\
(1) \\
\end{array}$} & \multirow{3}{*}{$\begin{array}{c}\text { Obs. Date } \\
(2)\end{array}$} & \multirow{3}{*}{$\begin{array}{c}\text { Telescope }^{a} \\
(3)\end{array}$} & \multicolumn{2}{|c|}{ Exposure Time (Section) } & \multirow{3}{*}{$\begin{array}{c}\text { Seeing } \\
\text { (arcseconds) } \\
(6)\end{array}$} & \multirow{3}{*}{$\begin{array}{c}\text { Source } \\
(7)\end{array}$} \\
\hline & & & $\mathrm{H} \alpha$ & $\mathrm{R}$ & & \\
\hline & & & (4) & $(5)$ & & \\
\hline NGC 0337 & 2001-10-19 & CTIO1.5 m & 1800 & 540 & 1.5 & SINGS \\
\hline NGC 1097 & $2001-10-17$ & CTIO1.5 m & 1800 & 480 & 1.1 & SINGS \\
\hline NGC 1512 & 2001-10-18 & CTIO1.5 m & 1800 & 450 & 1.2 & SINGS \\
\hline NGC 1566 & 2001-10-21 & CTIO1.5 m & 1800 & 540 & 1.3 & SINGS \\
\hline NGC 2403 & 2001-11-15 & $\mathrm{KPNO} 2.1 \mathrm{~m}$ & 1800 & 540 & 1.1 & SINGS \\
\hline NGC 3049 & 2002-04-11 & KPNO2.1 m & 1800 & 420 & 1.0 & SINGS \\
\hline NGC 3184 & $2002-04-15$ & KPNO2.1 m & 1800 & 840 & 1.3 & SINGS \\
\hline NGC 3198 & 2002-03-09 & $\mathrm{KPNO} 2.1 \mathrm{~m}$ & 1800 & 420 & 2.3 & SINGS \\
\hline NGC 3274 & 2001-03-03 & Bok & 1000 & 200 & 1.6 & LVL \\
\hline NGC 3344 & 2001-05-27 & Bok & 1000 & 200 & 1.3 & LVL \\
\hline NGC 3351 & 2001-03-29 & $\mathrm{KPNO} 2.1 \mathrm{~m}$ & 1800 & 360 & 1.1 & SINGS \\
\hline NGC 3368 & 2003-04-29 & VATT & 1200 & 120 & 1.5 & LVL \\
\hline NGC 3486 & $2001-05-27$ & Bok & 600 & 120 & 1.4 & LVL \\
\hline NGC 3521 & $2002-03-10$ & KPNO2.1 m & 1800 & 120 & 1.1 & SINGS \\
\hline NGC 3627 & 2002-04-14 & KPNO2.1 m & 1800 & 840 & 1.1 & SINGS \\
\hline NGC 4020 & 2001-03-03 & Bok & 1000 & 200 & 1.4 & LVL \\
\hline NGC 4051 & 2000-03-31 & JKT & 3600 & 600 & 1.5 & NED \\
\hline NGC 4136 & 2001-05-16 & JKT & 3600 & 300 & 1.0 & NED \\
\hline NGC 4288 & 2001-05-01 & Bok & 1000 & 200 & 1.3 & LVL \\
\hline NGC 4314 & $1996-02-13$ & INT & 1200 & 900 & 1.3 & NED \\
\hline NGC 4394 & 2001-02-17 & JKT & 3600 & 300 & 1.0 & NED \\
\hline NGC 4535 & 2000-03-06 & JKT & 3600 & 900 & 1.9 & NED \\
\hline NGC 4548 & $2000-03-31$ & JKT & 3600 & 600 & 1.7 & NED \\
\hline NGC 4569 & $1988-03-25$ & KPNO0.9 m & 3000 & 150 & 2.0 & NED \\
\hline NGC 4579 & 2002-03-10 & KPNO2.1 m & 1800 & 420 & 1.0 & SINGS \\
\hline NGC 4725 & $2002-03-11$ & KPNO2.1 m & 1800 & 840 & 1.6 & SINGS \\
\hline NGC 5248 & 2001-04-11 & JKT & 3600 & 1800 & 1.4 & NED \\
\hline NGC 5457 & $1995-05-20$ & KPNO Schmidt & 11700 & 9545 & 2.0 & NED \\
\hline NGC 5713 & 2001-03-30 & $\mathrm{KPNO} 2.1 \mathrm{~m}$ & 1800 & 420 & 1.5 & SINGS \\
\hline NGC 5832 & 2002-03-05 & Bok & 1000 & 200 & 2.1 & LVL \\
\hline NGC 6946 & 2001-03-31 & KPNO2.1 m & 1800 & 420 & 2.1 & SINGS \\
\hline NGC 7552 & 2001-10-17 & CTIO1.5 m & 1800 & 450 & 1.1 & SINGS \\
\hline NGC 7741 & $2000-11-10$ & JKT & 4800 & 600 & 1.5 & NED \\
\hline UGC 01249 & 2001-11-08 & Bok & 1000 & 200 & 1.7 & LVL \\
\hline
\end{tabular}

Notes. Col. (1): name of the galaxy. Col. (2): the date of the observation. Col. (3): the telescope used. Bok means the Steward Observatory Bok $2.3 \mathrm{~m}$ telescope on Kitt Peak; CTIO1.5 m means Cerro Tololo Inter-American Observatory $1.5 \mathrm{~m}$ telescope; INT means the $2.5 \mathrm{~m}$ Isaac Newton Telescope on La Palma; JKT means the $1 \mathrm{~m}$ Jacobus Kapteyn Telescope on La Palma; KPNO0.9 m means the Kitt Peak National Observatory $0.9 \mathrm{~m}$ telescope; KPNO2.1 m means the Kitt Peak National Observatory $2.1 \mathrm{~m}$ telescope; KPNO Schmidt means the Kitt Peak National Observatory Schmidt telescope; VATT means the Lennon $1.8 \mathrm{~m}$ Vatican Advanced Technology Telescope on Mt. Graham, AZ. Cols. (4) and (5): exposure time in each filter in units of seconds. Col. (6): seeing in arcseconds as measured on the reduced images. Col. (7): the sources where the data are archived. LVL: Dale et al. (2009), also part of Kennicutt et al. (2008). SINGS: Kennicutt et al. (2003). NED: the NASA Extragalactic Database (of which NGC 4051, NGC 4314, NGC 4535, NGC 4548, NGC 5248, and NGC 7741 are from Knapen et al. 2004 , NGC 4136 and NGC 4394 are from James et al. 2004, NGC 4569 is from Koopmann 2001, and NGC 5457 is from Hoopes et al. 2001).

morphology in many studies (e.g., Bershady et al. 2000; Conselice et al. 2000; Abraham et al. 2003; Gil de Paz et al. 2007; Muñoz-Mateos et al. 2009). The former is calculated by comparing the original image of a galaxy with its rotated counterpart (the usual rotation angle is $180^{\circ}$ ). Here, we followed the method in Conselice et al. (2000):

$$
A=\frac{\sum\left|I_{180^{\circ}}-I_{0}\right|}{2 \sum\left|I_{0}\right|},
$$

where $\mathrm{I}_{0}$ is the intensity distribution in the original image and $\mathrm{I}$ $180^{\circ}$ is the intensity distribution in the rotated image with a rotation angle of $180^{\circ}$.

The concentration index is usually calculated by the ratio of two radii containing fixed fractions of the total flux in a galaxy.
We adopt the index $\mathrm{C}_{42}$ defined by Kent (1985):

$$
C_{42}=5 \log _{10}\left(r_{80} / r_{20}\right) \text {, }
$$

where $r_{80}$ and $r_{20}$ are the semimajor axes of the ellipses containing $80 \%$ and $20 \%$ of the total luminosity, respectively. The resulting structural indices for the distributions of star formation and stellar mass in galaxies are listed in Table 4.

In Figure 6, we presented the results as a function of the bar strength $f_{\text {bar. }}$. The early- and late-type galaxies cover approximately the same range in $f_{\text {bar }}$, while they have different distributions in morphological parameter spaces. To determine the correlation between two variables, we perform Spearman's rho tests and the correlation coefficients for these are listed in each panel. The right panels of Figure 6 show the correlations between the stellar mass distribution and bar strength. In the whole sample, there are no clear trends between the 

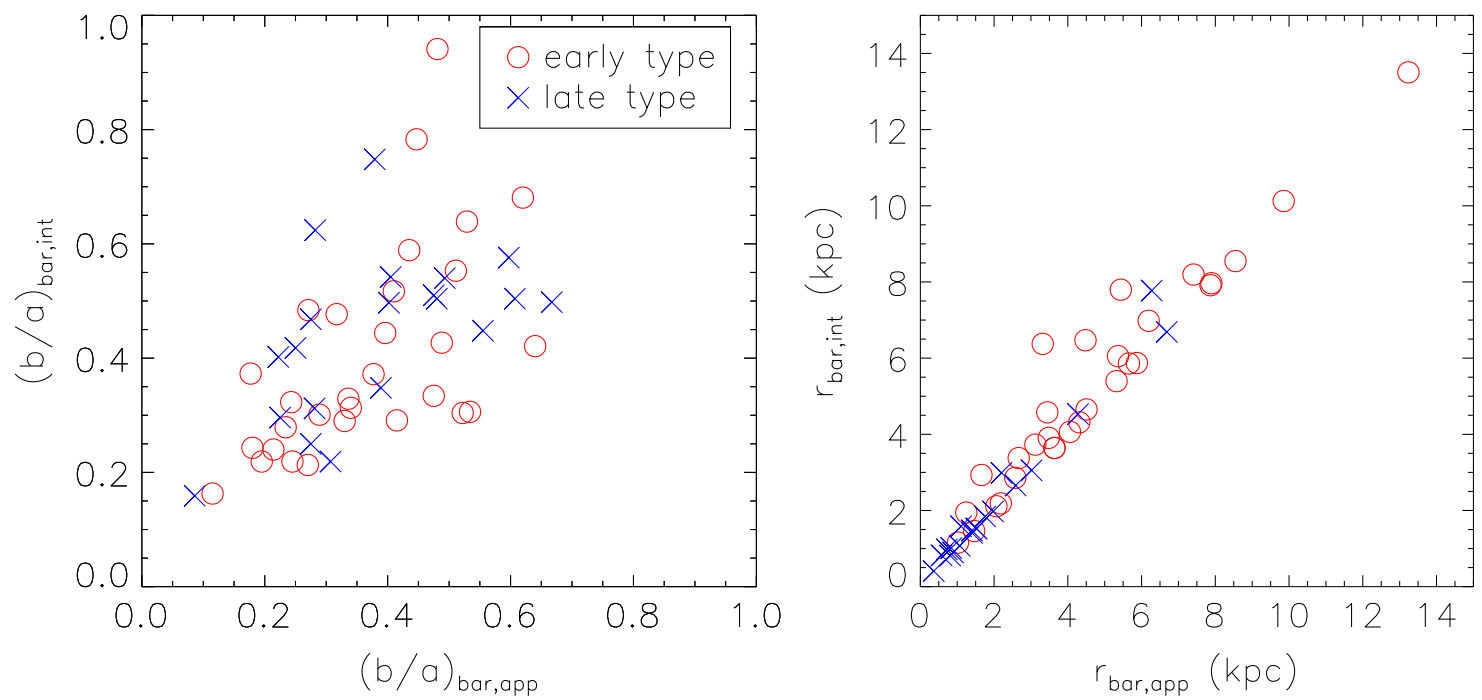

Figure 3. Comparison of bar properties between intrinsic and apparent values. The intrinsic axial ratio (b/a) bar,int $($ left $)$ and length $r_{\text {bar,int }}($ right $)$ of bars were calculated with the galaxy inclination and angles between the axes and line of nodes.

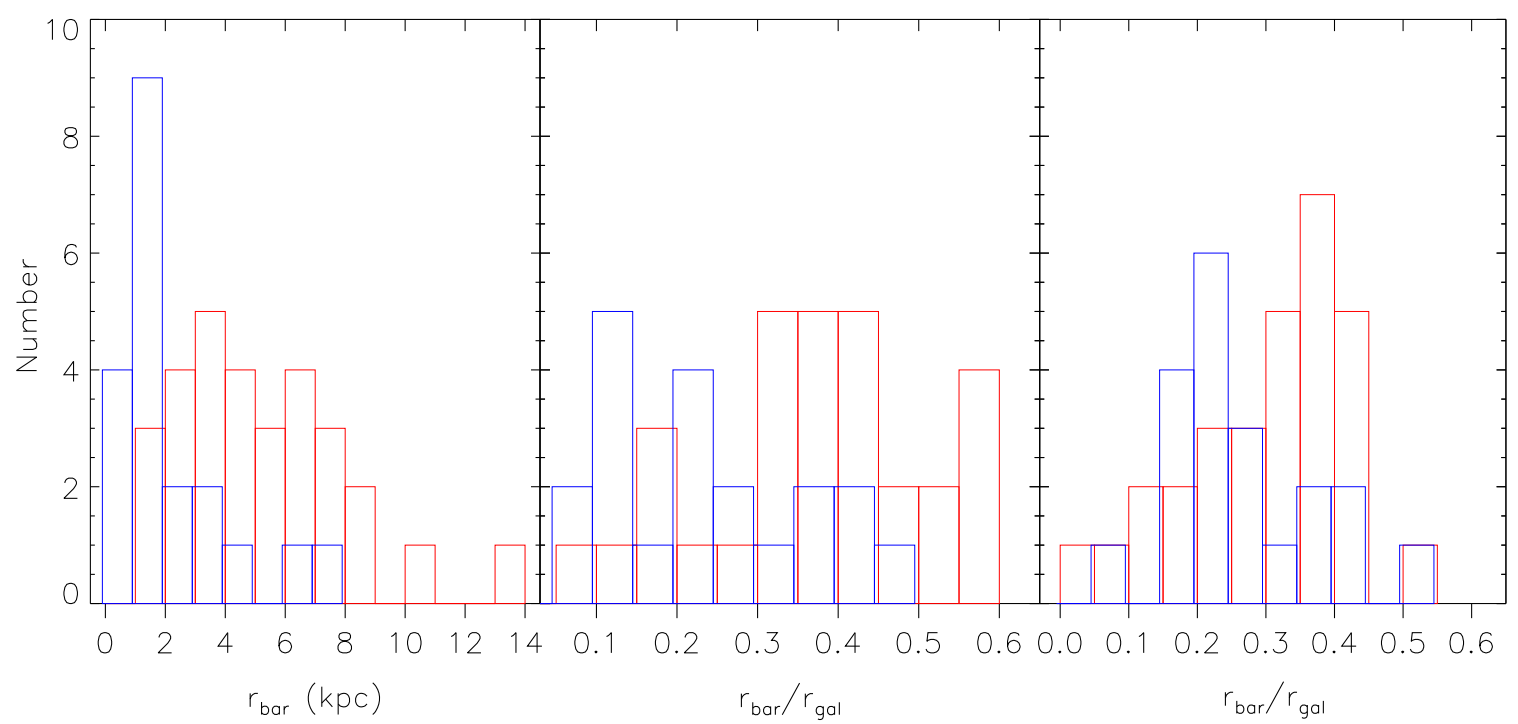

Figure 4. Distributions of bar properties of early- (red line) and late- (blue line) type spirals in our sample. The bar length (left) is indicated by the physical length of the bar semimajor axis, the normalized value (middle) is indicated by the ratio between the physical length of the bar semimajor axis and the galaxy radii at $25 \mathrm{mag} \operatorname{arcsec}^{-2}$, and the bar strength (right) is indicated by the ellipticity-based $f_{\text {bar }}$ parameter defined by Abraham \& Merrifield (2000). In each panel, two histograms are slightly offset in the $x$-axis for purposes of clarity.

morphological parameters $\left(A_{\text {mass }}\right.$ and $\left.C_{42, \text { mass }}\right)$ and bar strength $f_{\text {bar }}$ (Spearman's rho $r_{A}=-0.00$ and 0.30 , respectively). However, early-type galaxies show a higher concentration index and lower asymmetry than late-type ones, which was also found by previous studies (e.g., Muñoz-Mateos et al. 2009). The results presented here show a downsizing signature of the formation of galactic structures (Cowie et al. 1996; Bundy 2006; Sheth et al. 2008), i.e., more concentrated galaxies mean more mature systems.

The left panels of Figure 6 present the variation in the distribution of star formation activity with bar strength. No clear trend is found between them (Spearman's rho $r_{A}=-0.12$ and 0.30 , respectively). In general, star formation activity in early-type galaxies is less asymmetric and more concentrated than in late-type samples, although it is not always well distinguished. There are tens of early-type galaxies that also have $A_{\mathrm{SFR}}$ or $C_{\mathrm{SFR}}$ similar to those of late-type ones, and most early-type galaxies with high $C_{\mathrm{SFR}}$ are located in the high end of the bar strength. In addition, weaker bars have lower concentric star formation activity in all types of galaxies, while the star formation activity in the spirals with stronger bars does not always have a high concentric distribution, especially in early-type galaxies. This indicates that galaxies with similar bars can have differing distributions of star formation activities.

\subsection{Global Star Formation and Star Formation in Different Structural Components}

In order to further explore the possible relation between star formation activity and stellar bars, we investigate the correlation between $f_{\text {bar }}$ and SFRs, SFR surface densities $\left(\Sigma_{\mathrm{SFR}}\right)$, and 

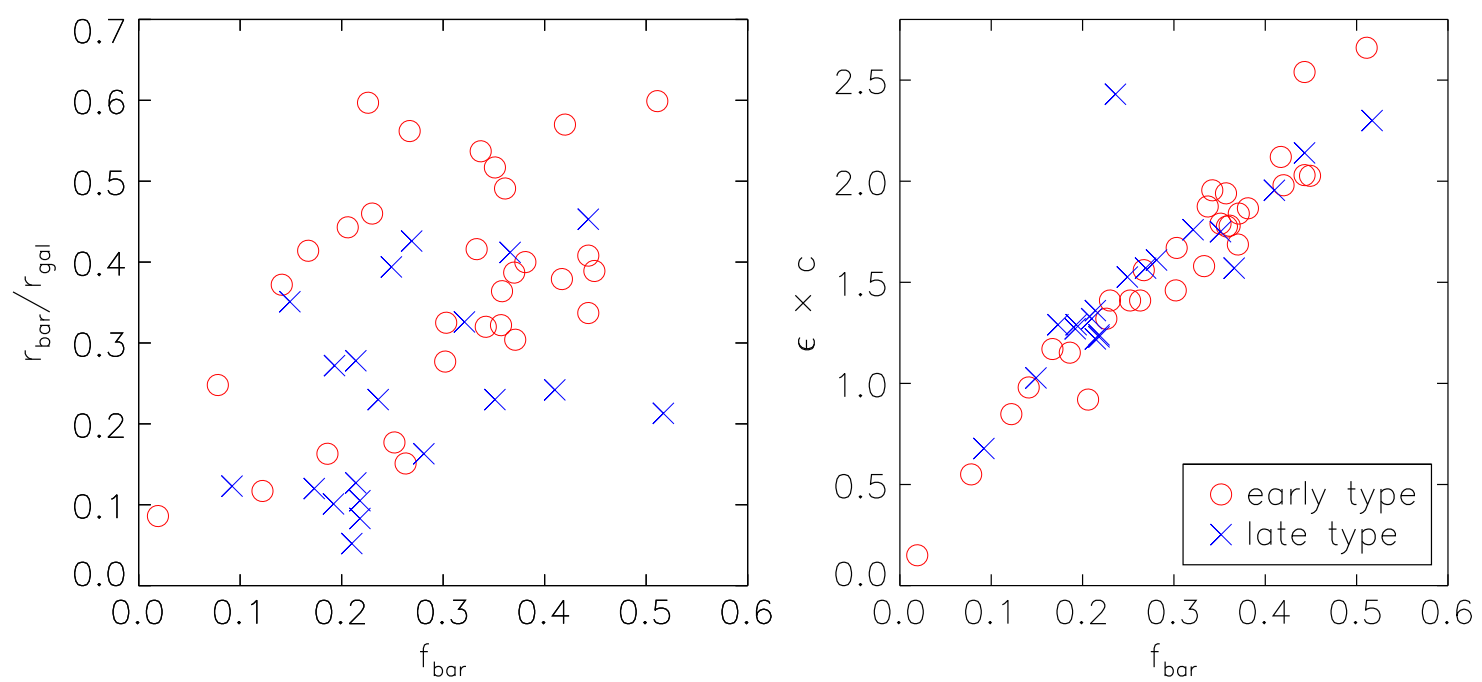

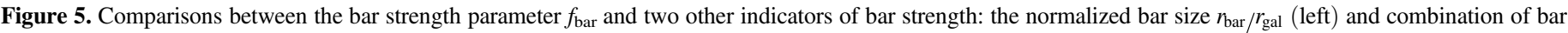
ellipticity and boxiness $\epsilon \times c$ (right). The symbols are the same as in the right panel of Figure 2 .

SSFRs in different galactic structural components including bulges, bars, and global galaxies. The regions of these structures are defined based on the decomposition results of the $3.6 \mu \mathrm{m}$ images (see Section 3). Each galaxy is divided into three structural components: bulge, bar, and outer disk. In one galaxy, three structures have no overlap regions, i.e., the outer disk region is obtained by subtracting the bar and bulge field in the galaxy, and the bar region is obtained by subtracting its central bulge field. The bulge regions are defined following the decomposition results or the galactic central kiloparsec regions if no bulges exist in the decomposition fitting. The inclination $i$ of galaxies can lead to underestimating the projected area of each galactic structure, and may affect the estimate of $\Sigma_{\text {SFR }}$ (SFR/area). Therefore, we correcte this variation included by the inclination using the method in Martin (1995; see Section 3 ), and then calculate $\Sigma_{\mathrm{SFR}}$. The results are listed in Table 4, and are plotted in Figure 7.

First, the top panel of Figure 7 shows the SFRs as a function of the bar strength $f_{\mathrm{bar}}$. There are no clear trends for the SFRs in each structure with bar strength, although weak trends are found where the SFRs in bulges increase toward higher bar strength along with large scatter (Spearman's rho $r_{A}=0.29$ ), similar to the result in Ellison et al. (2011). Another finding is that the early- and late-type galaxies have different behaviors in the plot: (1) early-type galaxies have higher SFRs in each regions on average and (2) late-type ones vary more in their star formation properties, especially for intermediate-strength bars $\left(f_{\mathrm{bar}} \sim 0.2-0.4\right)$. This may indicate that star formation is not a simple function of bar strength or bar-related factors, and it likely also depends on other factors (Sheth et al. 2002).

Next, we compare the SFR densities and bar strength in the middle panel of Figure 7. Similar to SFRs, the correlation coefficients are less than 0.2 , indicating no clear trend of $\Sigma_{\mathrm{SFR}}$ with $f_{\text {bar. }}$. In addition, there is no significant difference between early- and late-type galaxies in the distribution of $\Sigma_{\text {SFR }}$. This suggests that galaxies of both types have similar $\Sigma_{\mathrm{SFR}}$, although their SFRs are different.

Last, we show the correlation between SSFR and bar strength in the bottom panel of Figure 7. There is a remarkable difference between the two types of galaxies: most late-type samples have SSFRs higher than $0.1 \mathrm{Gyr}^{-1}$, while most earlytype ones have SSFRs lower than $0.1 \mathrm{Gyr}^{-1}$. This trend is remarkable in all regions except for bulges. Bulges have the largest scatter in the distribution of SSFRs; the highest SSFR is $\sim 10 \mathrm{Gyr}^{-1}$ in one late-type galaxy (NGC 4654), while the lowest value is nearly four orders of magnitude lower than it.

\section{DISCUSSION}

A series of analyses has been provided in this work to explore the correlations between stellar bars and star formation activities in galaxies, both sub-structures and galaxies as a whole. The analysis of these correlations constrains the effect of galactic bars to star formation and reveals secular evolution to be a complex process in barred galaxies rather than a simple one as previously assumed. In this section, we aim to discuss the possible reasons for star formation activity varying along stellar bars. Specifically, we determine how the behaviors connect to possible episodes of a bar-driven secular process.

\subsection{The Impact of Bars on Star Formation Activity}

For barred galaxies in our sample, we had expected some evidence for enhanced star formation activity toward stronger bars. However, we find that there are no clear trends for the star formation properties with bar strength, except for a weak dependence on the bars for SFRs and $\Sigma_{\text {SFR }}$ in the bulges and global galaxies. Some previous studies also derived similar results. Using a control sample of disk galaxies, Ellison et al. (2011) compared SFRs between barred and unbarred galaxies and found that there is no correlation between bar length or bar axial ratio and the enhancement of the SFR. Some earlier studies using far-infrared emission as a proxy for SFR also did not find definite evidence that stronger bars could result in higher SFRs (e.g., Pompea \& Rieke 1990; Isobe \& Feigelson 1992; Roussel et al. 2001).

On the other hand, simulations indeed show that gas inflows driven by bars can enhance star formation activity in galactic central regions (e.g., Athanassoula et al. 2009). Gas being transported to the center by bars is also found in observations 
Table 4

Structural and Star Formation Properties of the Sample

\begin{tabular}{|c|c|c|c|c|c|c|c|c|c|c|c|c|c|c|c|}
\hline \multirow[b]{2}{*}{$\begin{array}{l}\text { Name } \\
\text { (1) }\end{array}$} & \multirow[b]{2}{*}{$\begin{array}{l}f_{\mathrm{bar}} \\
(2)\end{array}$} & \multirow[b]{2}{*}{$\begin{array}{c}C_{42, \mathrm{SFR}} \\
(3)\end{array}$} & \multirow[b]{2}{*}{$\begin{array}{c}A_{\mathrm{SFR}} \\
(4)\end{array}$} & \multirow[b]{2}{*}{$\begin{array}{c}C_{42 \text {,mass }} \\
(5)\end{array}$} & \multirow[b]{2}{*}{$\begin{array}{c}A_{\text {mass }} \\
(6)\end{array}$} & \multicolumn{3}{|c|}{$\log \operatorname{SFR}\left(M_{\odot} \mathrm{yr}^{-1}\right)$} & \multicolumn{3}{|c|}{$\log \Sigma_{\mathrm{SFR}}\left(M_{\odot} \mathrm{yr}^{-1} \mathrm{kpc}^{-2}\right)$} & \multicolumn{3}{|c|}{$\log \operatorname{SSFR}\left(\mathrm{Gyr}^{-1}\right)$} & \multirow{2}{*}{$\begin{array}{l}\text { Stage } \\
\text { (16) }\end{array}$} \\
\hline & & & & & & $\begin{array}{c}\text { Global } \\
(7)\end{array}$ & $\begin{array}{c}\text { Bulge } \\
(8)\end{array}$ & $\begin{array}{c}\text { Bar } \\
(9)\end{array}$ & $\begin{array}{c}\text { Global } \\
(10)\end{array}$ & $\begin{array}{c}\text { Bulge } \\
(11)\end{array}$ & $\begin{array}{l}\text { Bar } \\
(12)\end{array}$ & $\begin{array}{c}\text { Global } \\
\text { (13) }\end{array}$ & $\begin{array}{c}\text { Bulge } \\
(14)\end{array}$ & $\begin{array}{c}\text { Bar } \\
(15)\end{array}$ & \\
\hline NGC 0023 & 0.23 & 3.62 & 0.31 & 4.33 & 0.06 & 1.06 & 0.79 & 0.58 & -1.91 & -0.17 & -1.35 & -1.08 & -0.90 & -0.95 & $\mathrm{c}$ \\
\hline NGC 0266 & 0.34 & 1.92 & 0.71 & 4.40 & 0.08 & 0.62 & -0.64 & -0.08 & -2.67 & -1.50 & -2.34 & -1.72 & -2.00 & -1.88 & $\mathrm{~b}$ \\
\hline NGC 0337 & 0.25 & 2.43 & 0.54 & 2.58 & 0.21 & 0.20 & -1.10 & -0.39 & -2.06 & -1.00 & -1.46 & -0.66 & -0.57 & -0.61 & $\mathrm{~b}$ \\
\hline NGC 1097 & 0.38 & 5.50 & 0.28 & 5.38 & 0.11 & 0.58 & 0.23 & -0.26 & -2.54 & -0.53 & -1.98 & -1.39 & -1.15 & -1.45 & $\mathrm{~d}$ \\
\hline NGC 1512 & 0.37 & 5.15 & 0.62 & 4.38 & 0.07 & -0.22 & -0.92 & -1.27 & -3.08 & -0.91 & -2.53 & -1.34 & -1.03 & -1.67 & $\mathrm{~d}$ \\
\hline NGC 1566 & 0.26 & 2.27 & 0.51 & 3.88 & 0.11 & 0.83 & -0.64 & -0.35 & -2.45 & -0.57 & -1.60 & -1.21 & -1.34 & -1.47 & $\mathrm{c}$ \\
\hline NGC 2403 & 0.22 & 1.89 & 0.47 & 3.37 & 0.25 & 0.24 & -1.90 & -0.88 & -2.04 & -0.68 & -0.87 & -0.09 & -0.03 & -0.05 & $\mathrm{~b}$ \\
\hline NGC 2903 & 0.52 & 3.49 & 0.42 & 3.18 & 0.10 & 0.59 & -0.54 & -0.18 & -2.56 & -0.11 & -1.17 & -1.29 & -0.81 & -1.03 & $\mathrm{c}$ \\
\hline NGC 2964 & 0.36 & 2.50 & 0.32 & 3.16 & 0.12 & 0.59 & -0.41 & -0.25 & -1.82 & 0.06 & -1.15 & -0.77 & -0.54 & -0.75 & $\mathrm{c}$ \\
\hline NGC 3049 & 0.51 & 4.16 & 0.57 & 4.06 & 0.13 & -0.05 & -0.27 & -0.68 & -2.33 & -0.40 & -1.67 & -0.74 & 0.07 & -0.65 & $\mathrm{c}$ \\
\hline NGC 3184 & 0.17 & 1.57 & 0.61 & 2.45 & 0.15 & 0.06 & -1.57 & -1.63 & -2.62 & -1.06 & -2.19 & -1.16 & -0.74 & -1.36 & $\mathrm{c}$ \\
\hline NGC 3198 & 0.09 & 2.02 & 0.46 & 2.79 & 0.11 & -0.00 & -1.46 & -1.17 & -2.85 & -0.66 & -2.06 & -1.00 & -0.63 & -1.09 & $\mathrm{c}$ \\
\hline NGC 3274 & 0.21 & 1.18 & 0.56 & 2.75 & 0.12 & -0.85 & -1.89 & -1.64 & -2.44 & -1.17 & -1.76 & -0.38 & -0.21 & -0.38 & $\mathrm{~b}$ \\
\hline NGC 3319 & 0.28 & 2.34 & 0.74 & 3.16 & 0.09 & -0.69 & -3.70 & -1.68 & -3.36 & -2.35 & -2.37 & -1.05 & -1.24 & -0.99 & $\mathrm{~b}$ \\
\hline NGC 3344 & 0.12 & 2.30 & 0.72 & 2.96 & 0.11 & 0.18 & -1.53 & -1.37 & -2.51 & -0.27 & -2.03 & -1.03 & -0.76 & -1.57 & $\mathrm{~d}$ \\
\hline NGC 3351 & 0.37 & 5.86 & 0.30 & 4.60 & 0.05 & 0.52 & -0.03 & -0.54 & -2.42 & -0.71 & -2.07 & -1.23 & -1.01 & -1.45 & $\mathrm{~d}$ \\
\hline NGC 3368 & 0.33 & 3.33 & 0.41 & 4.55 & 0.05 & 0.32 & -0.47 & -0.14 & -2.72 & -1.17 & -1.91 & -1.73 & -1.75 & -1.64 & $\mathrm{~b}$ \\
\hline NGC 3486 & 0.19 & 2.50 & 0.59 & 4.00 & 0.11 & 0.04 & -2.25 & -1.45 & -2.74 & -1.65 & -1.95 & -0.99 & -1.83 & -1.41 & $\mathrm{~b}$ \\
\hline NGC 3521 & 0.02 & 2.24 & 0.29 & 3.70 & 0.07 & 1.02 & -1.12 & -0.06 & -2.29 & -0.78 & -1.19 & -1.26 & -1.87 & -1.40 & $\mathrm{~b}$ \\
\hline NGC 3627 & 0.30 & 2.62 & 0.63 & 3.50 & 0.16 & 1.07 & -0.57 & 0.15 & -2.00 & -0.76 & -1.37 & -1.06 & -1.43 & -1.22 & $\mathrm{~b}$ \\
\hline NGC 4020 & 0.15 & 1.50 & 0.55 & 2.23 & 0.13 & -0.78 & -3.05 & -1.43 & -2.55 & -1.78 & -2.09 & -0.76 & -0.73 & -0.74 & $\mathrm{~b}$ \\
\hline NGC 4051 & 0.42 & 7.55 & 0.38 & 6.96 & 0.18 & 0.34 & -0.22 & -0.80 & -2.12 & -0.51 & -1.71 & -0.83 & -0.78 & -0.74 & $\mathrm{~d}$ \\
\hline NGC 4136 & 0.21 & 1.95 & 0.59 & 2.81 & 0.10 & -0.07 & -2.64 & -2.19 & -2.26 & -1.82 & -2.25 & -0.37 & -1.03 & -1.14 & $\mathrm{~b}$ \\
\hline NGC 4288 & 0.32 & 1.70 & 0.63 & 2.63 & 0.14 & -1.04 & -1.92 & -2.47 & -2.52 & -1.82 & -1.99 & -0.44 & -0.52 & -0.05 & $\mathrm{~b}$ \\
\hline NGC 4303 & 0.25 & 1.44 & 0.52 & 2.97 & 0.18 & 1.34 & -0.17 & 0.10 & -1.94 & -0.03 & -1.30 & -0.96 & -0.95 & -1.20 & $\mathrm{c}$ \\
\hline NGC 4314 & 0.42 & 4.19 & 0.40 & 4.20 & 0.04 & -0.21 & -0.46 & -0.84 & -2.74 & -0.80 & -2.22 & -1.67 & -1.07 & -1.76 & $\mathrm{c}$ \\
\hline NGC 4394 & 0.44 & 6.05 & 0.65 & 4.52 & 0.06 & -0.15 & -0.79 & -1.19 & -2.55 & -0.72 & -2.10 & -1.29 & -1.00 & -1.53 & $\mathrm{~d}$ \\
\hline NGC 4491 & 0.17 & 5.38 & 0.59 & 2.93 & 0.06 & -1.69 & -2.09 & -2.16 & -3.08 & -1.15 & -2.53 & -1.50 & -0.37 & -1.50 & $\mathrm{~d}$ \\
\hline NGC 4535 & 0.41 & 1.22 & 0.75 & 2.48 & 0.14 & 0.15 & -0.78 & -1.41 & -3.36 & -1.21 & -3.06 & -2.04 & -1.39 & -2.34 & $\mathrm{~d}$ \\
\hline NGC 4548 & 0.45 & 1.70 & 0.31 & 3.83 & 0.07 & -0.32 & -1.67 & -1.74 & -2.69 & -1.99 & & -1.47 & -1.92 & & $\mathrm{~b}$ \\
\hline NGC 4569 & 0.08 & 3.07 & 0.42 & 3.86 & 0.09 & 0.32 & -0.47 & -0.00 & -2.93 & -1.13 & -1.90 & -1.54 & -1.34 & -1.30 & $\mathrm{~b}$ \\
\hline NGC 4579 & 0.30 & 4.06 & 0.51 & 4.29 & 0.05 & 0.58 & -0.21 & -0.25 & -2.59 & -0.87 & -1.97 & -1.72 & -1.50 & -1.86 & $\mathrm{c}$ \\
\hline NGC 4580 & 0.14 & 1.31 & 0.42 & 2.53 & 0.06 & -0.52 & -1.83 & -0.71 & -2.52 & -1.48 & -1.64 & -1.41 & -1.07 & -1.03 & $\mathrm{~b}$ \\
\hline NGC 4647 & 0.22 & 2.50 & 0.32 & 2.98 & 0.33 & 0.37 & -1.43 & -1.13 & -2.12 & -0.83 & -1.03 & -0.99 & -0.68 & -0.81 & $\mathrm{~b}$ \\
\hline NGC 4654 & 0.24 & 6.24 & 0.46 & 2.90 & 0.18 & 0.82 & 0.38 & 0.37 & -1.92 & 0.70 & -0.75 & -0.66 & 0.87 & -0.27 & $\mathrm{~d}$ \\
\hline NGC 4725 & 0.34 & 1.60 & 0.56 & 3.96 & 0.09 & 0.52 & -1.05 & -0.70 & -2.97 & -2.19 & -2.66 & -1.69 & -2.36 & -2.04 & $\mathrm{~b}$ \\
\hline NGC 5248 & 0.19 & 7.62 & 0.53 & 4.02 & 0.16 & 1.03 & 0.73 & -0.13 & -1.96 & 0.50 & -1.22 & -0.70 & 0.08 & -1.00 & d \\
\hline NGC 5377 & 0.23 & 1.31 & 0.29 & 4.49 & 0.05 & 0.53 & -1.20 & -0.40 & -2.28 & -1.84 & -2.43 & -1.00 & -1.92 & -1.57 & $\mathrm{~b}$ \\
\hline NGC 5457 & 0.21 & 2.64 & 0.68 & 2.82 & 0.22 & 0.44 & -2.02 & -1.44 & -2.69 & -0.54 & -1.70 & -0.81 & -0.47 & -1.05 & c \\
\hline NGC 5713 & 0.44 & 3.23 & 0.36 & 3.41 & 0.24 & 0.68 & -0.15 & 0.09 & -1.94 & -0.59 & -0.80 & -1.04 & -0.79 & -0.80 & $\mathrm{~b}$ \\
\hline NGC 5832 & 0.27 & 1.95 & 0.68 & 2.61 & 0.08 & -1.53 & -4.78 & -2.00 & -3.11 & -3.10 & -2.70 & -0.90 & -1.74 & -1.00 & $\mathrm{a}$ \\
\hline NGC 6701 & 0.35 & 4.16 & 0.38 & 4.38 & 0.17 & 0.95 & 0.32 & 0.58 & -1.68 & -0.23 & -0.94 & -0.96 & -0.86 & -0.83 & $\mathrm{e}$ \\
\hline NGC 6946 & 0.35 & 2.85 & 0.56 & 2.98 & 0.26 & 0.72 & -0.53 & -0.30 & -1.90 & 0.01 & -1.12 & -0.90 & -0.60 & -0.74 & $\mathrm{c}$ \\
\hline NGC 7080 & 0.36 & 4.16 & 0.39 & 4.67 & 0.14 & 0.79 & 0.08 & 0.04 & -2.13 & -0.58 & -1.69 & -1.16 & -1.04 & -1.17 & $\mathrm{c}$ \\
\hline NGC 7479 & 0.37 & 3.65 & 0.45 & 3.88 & 0.16 & 0.86 & -0.08 & 0.18 & -2.06 & -0.38 & -1.41 & -0.97 & -0.82 & -1.00 & c \\
\hline NGC 7552 & 0.21 & 5.38 & 0.25 & 5.53 & 0.09 & 0.74 & 0.41 & 0.26 & -1.68 & 0.42 & -1.15 & -0.92 & -0.72 & -0.89 & d \\
\hline NGC 7591 & 0.36 & 3.52 & 0.29 & 4.00 & 0.08 & 0.93 & 0.05 & 0.41 & -2.05 & -0.21 & -1.16 & -0.95 & -0.95 & -0.88 & $\mathrm{~b}$ \\
\hline NGC 7741 & 0.44 & 2.53 & 0.49 & 2.73 & 0.18 & -0.91 & -2.26 & -1.65 & -2.50 & -1.52 & -1.84 & -0.53 & -0.29 & -0.49 & $\mathrm{~b}$ \\
\hline NGC 7798 & 0.19 & 5.04 & 0.54 & 3.53 & 0.14 & 0.82 & -0.01 & 0.43 & -1.18 & 0.27 & -0.11 & -0.32 & -0.15 & 0.17 & $\mathrm{e}$ \\
\hline UGC 1249 & 0.27 & 2.15 & 0.72 & 2.61 & 0.18 & -2.92 & -4.00 & -3.30 & -3.38 & -2.13 & -2.60 & -0.37 & -0.00 & -0.08 & $\mathrm{~b}$ \\
\hline
\end{tabular}

Notes. (1) Galaxy name. (2) Bar strength estimated using Equation (1). (3) and (4) The concentration and asymmetry index for the star formation distribution in one galaxy, described in Section 4.1. (5) and (6) The concentration and asymmetry index for the stellar mass distribution in one galaxy, described in Section 4.1. (7)-(15) The star formation rate, surface density of star formation rate, and specific star formation rate for the galaxy as an entirety, bulge and bar, respectively, calculated as in Section 4.2. (16) The stage in bar-driven evolutionary sequence, estimated from the position in Figure 9 and described in Section 5.2.

(Regan et al. 1995, 1999; Sheth et al. 2000; Zurita and Pérez 2008). In addition, simulations prove that the efficiency of driving gas depends on bar strength, i.e., stronger bars are able to transport more material at a faster rate than weak bars (e.g., Athanassoula 1992; Regan \& Teuben 2004).
When focusing on different types of galaxies, we find that early-type galaxies have higher SFRs, but similar $\Sigma_{\mathrm{SFR}}$ and lower SSFRs compared with late-type galaxies. This may be due to the differing episodes of bar-driven gas inflow and star formation in galaxies with different types. In different episodes, 


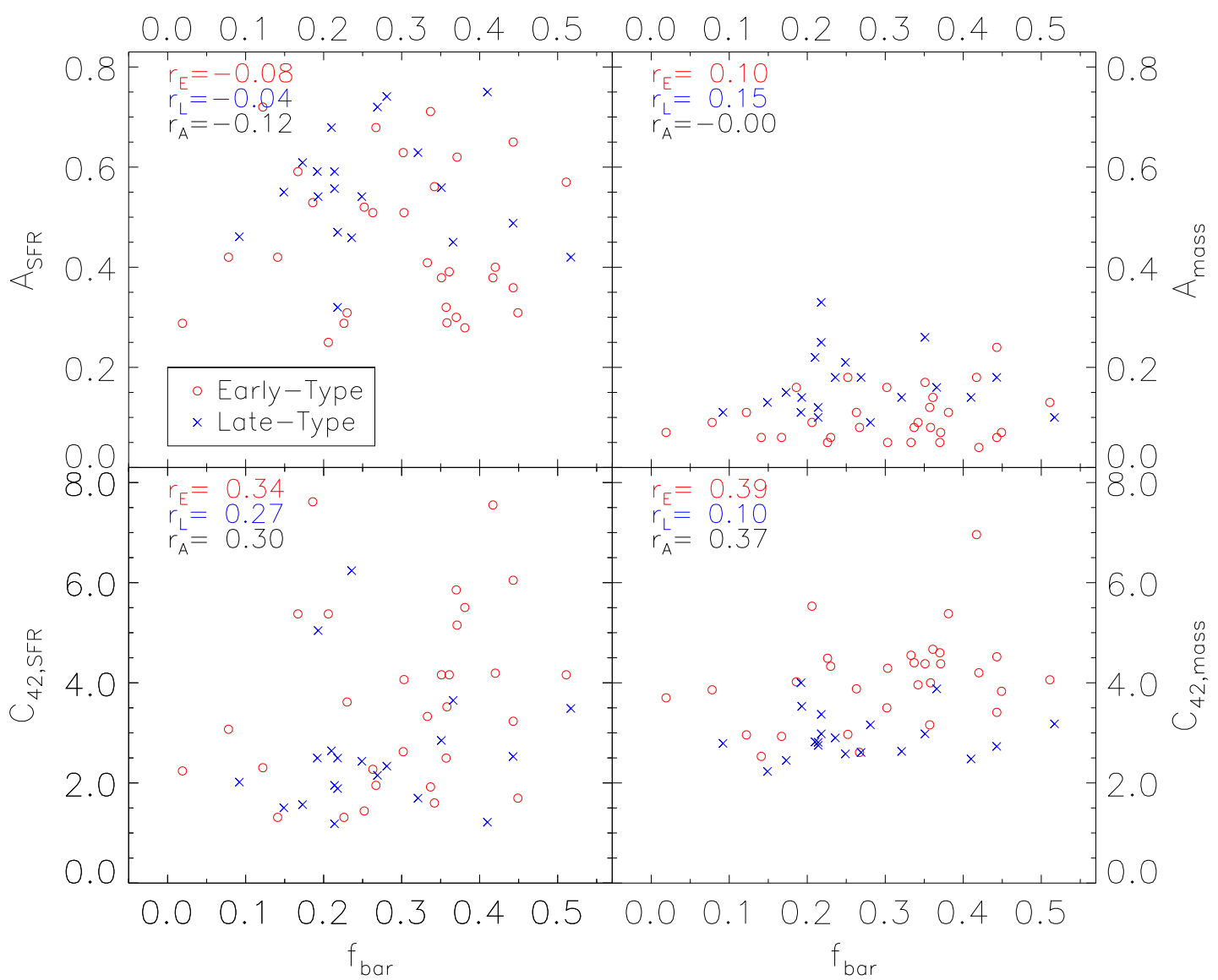

Figure 6. Relationship between bar strength and asymmetry (upper panels) and concentration index (lower panels) in our samples, derived based on the SFR images (left) and mass images (right) in Figure 1. In each panel, Spearman's rho tests are made to test the correlation between two variables, and the correlation coefficients are listed for the early-type galaxies $\left(r_{E}\right)$, late-type galaxies $\left(r_{L}\right)$, and the whole sample $\left(r_{A}\right)$ of galaxies. The four panels use the same symbols: red circles for earlytype galaxies and blue crosses for late-type ones.

there are likely differing bar stages, bulge mass, gas contents, and other physical conditions, all of which may contribute to this process (Coelho \& Gadotti 2011; Ellison et al. 2011; Oh et al. 2012; Melvin et al. 2014). In late-type galaxies, stellar bars tend to be short, and bulges are usually small if they exist. This is likely the early stage of bar-driven evolution. In this episode, the effect of bars is not pronounced, although there is sufficient gas in galactic disks. Within the process, the gas is reserved in the bar and galactic central regions, and then star formation activity takes place there, resulting in the growth of the stellar bar and bulge along with a change in the galaxy morphology. Therefore, we can expect to find "mature" bars in early-type disks (Debattista et al. 2006; Giordano et al. 2011).

However, the various trends of star formation properties with bar strength suggest that another process is at play in reducing the bar effect: (1) massive bulges can dilute the bar nonaxisymmetric forces (Das et al. 2003; Laurikainen et al. 2004), thus some early-type spirals may have weaker bars than late-type systems (e.g., Laurikainen et al. 2002), (2) the amount of gas content may be not sufficient after being consumed by star formation in the previous process. Using CO observations, Sheth et al. (2005) found that six early-type barred spirals have very little molecular gas detected in bars and nuclear regions due to this gas being consumed during star formation. Therefore, early-type systems may have $\Sigma_{\mathrm{SFR}}$ in their bars and central regions that are similar to or lower than those of late-type ones (Figure 7). Furthermore, these probabilities are likely the reasons why various star formation properties are associated with the intermediate-strength bars. Some of these bars may be transitioning from weak to strong and others with similar strength may have been weakened by massive bulges, although could have been strong once. Besides these factors, the difference between the timescales of bar evolution and star formation also can contribute to the complexity of the process (Roussel et al. 2001). It is likely that the lifetime of large-scale stellar bars is long enough to allow multiple episodes of central star formation, which can be used to interpret why some early-type bulges have lower SSFRs (Figure 7).

\subsection{Bar-driven Secular Evolution}

As mentioned above, bar-driven secular evolution is a complex process and is likely composed of a series of stages. There has been substantial progress in scenarios of secular evolution based on simulation evidence and observational results (e.g., Martin \& Friedli 1997; Combes 2000; Bournaud $\&$ Combes 2002).

In the evolution process, numerous episodes of bar-driven gas inflow and star formation may take place, along with the growth, destruction, and re-formation of stellar bars (Combes 2009). 


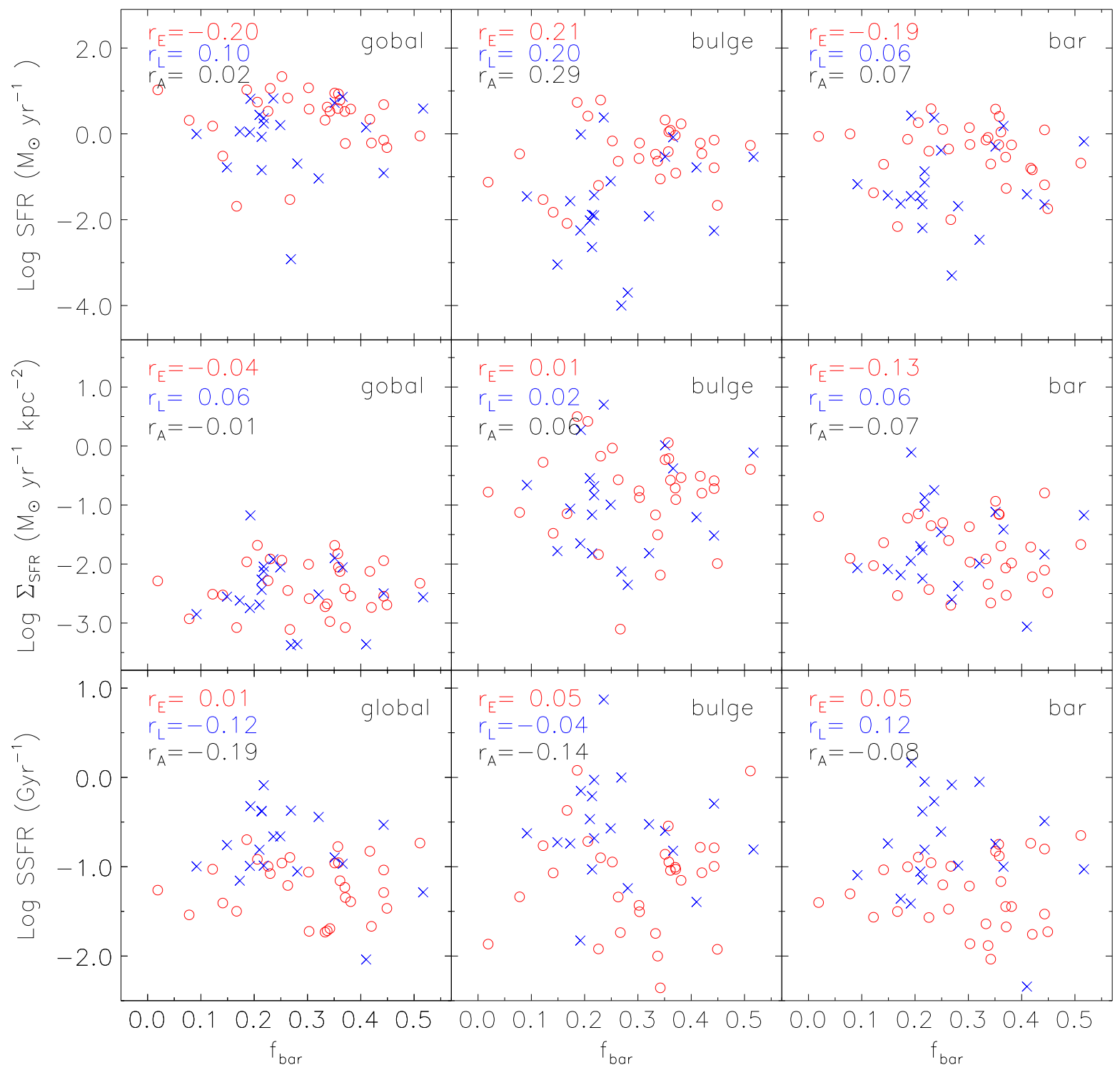

Figure 7. Relationship between bar strength and star formation activity in galaxies. From top to bottom, bar strength is compared with SFR, $\Sigma_{\text {SFR }}$, and SSFR in the global galaxy (left), the bulge (middle), and the bar (right), respectively. Spearman's rho tests are made as in Figure 6 . The symbols are the same as in Figure 2.

In one simple scenario, various stages of evolution can also be divided based on the changing properties of star formation activity and gas content in galaxies. Using properties of $\mathrm{H} \alpha$ emission in barred galaxies, Verley et al. (2007) classified such galaxies into three main groups, in which $\mathrm{H} \alpha$ emission is mainly located in stellar bars, galactic central regions, and spiral arms. They suggested that these groups are different stages of an evolution process, similar to the trend proposed by Martin \& Friedli (1997), but more detailed. Based on the properties of circumnuclear gas and star formation, Jogee et al. (2005) projected a possible picture of bar-driven dynamical evolution where the evolution process of barred galaxies was divided into three stages (i.e., Type I non-starburst, Type II nonstarburst, and circumnuclear starburst). In the three stages, large amounts of gas are transformed from stellar bars to galactic central regions step by step along with the star formation activity in bars and central regions. To complement this picture, Sheth et al. (2005) supplemented a post-starburst phase, i.e., Type III non-starburst, where the gas has been consumed by a circumnuclear starburst with no molecular gas in the bar region.

Given the above evolution pictures, molecular gas is transported from galactic disks to bulges through stellar bars, resulting in the rearrangement of material in the three galactic structures. Since star formation activity is correlated with the local gas density (Kennicutt 1998), the properties of star formation activity in bulges are likely associated with those in bars and disks. In addition, star formation activity in barred galaxies, especially in bulges, can change the galactic morphological properties (e.g., the asymmetry and concentration). To explore the correlations between them, we compared the star formation parameters of bulges, bars, and disks in our sample.

Figure 8 illustrates the correlations between the SFRs, $\Sigma_{\mathrm{SFR}}$, and SSFRs in three galactic structures, along with the ratio lines from $10^{-2}$ to $10^{2}$ plotted. We find that barred galaxies with more active star formation in bars tend to have more active star formation in bulges with a similar correlation between bars 


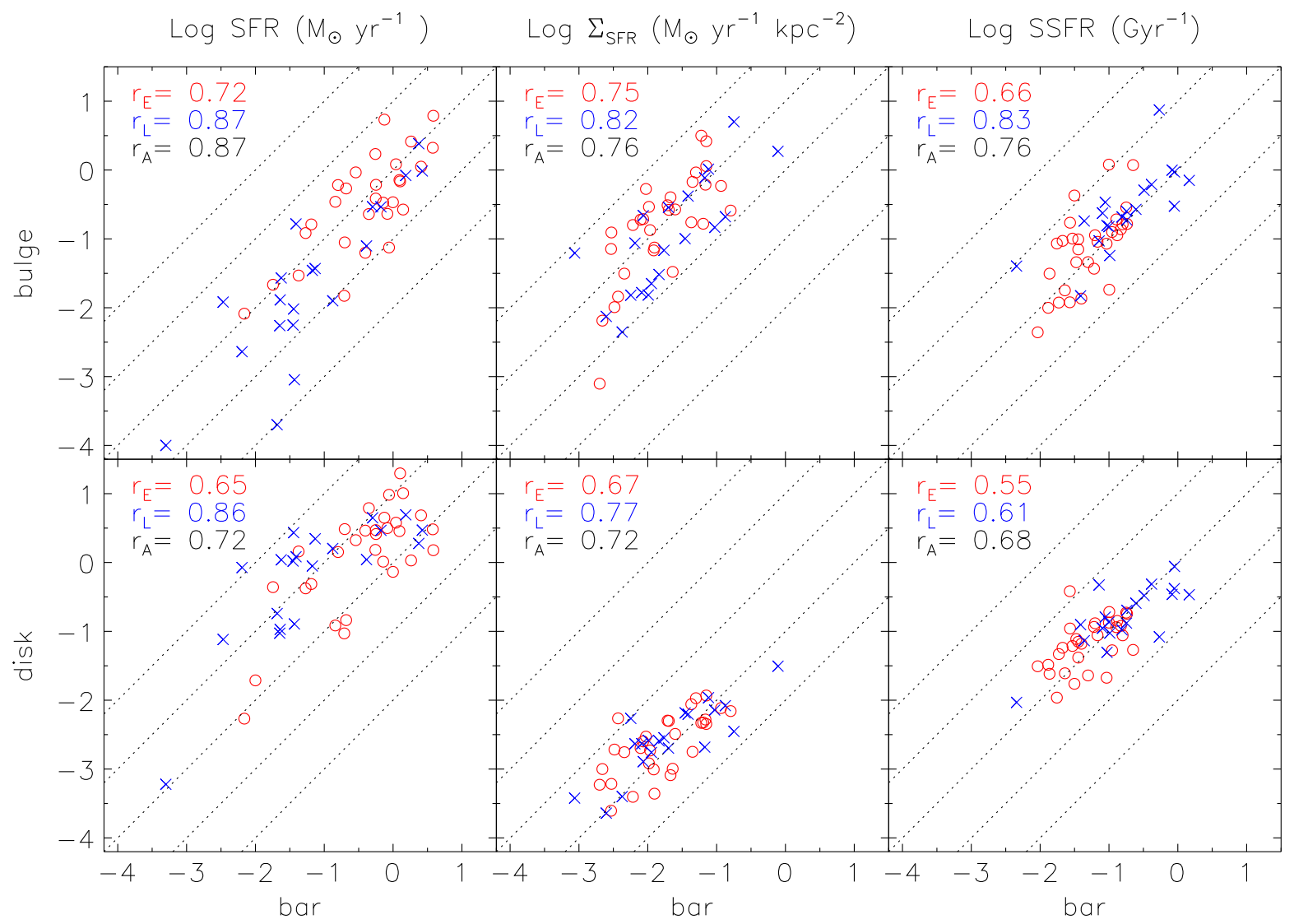

Figure 8. Comparison of the SFR (left), $\Sigma_{\mathrm{SFR}}$ (middle), and SSFR (right) in different structures: bar vs. bulge (top) and bar vs. disk (bottom). The dotted lines in each panel mark the scales between the two structures from $10^{-2}$ to $10^{2}$, top to bottom, with steps of one order of magnitude. The symbols are the same as in Figure 2 .

and disks. It is worth noting that most SFRs in bars are 0.1-10 times those in bulges, while the ratios of $\Sigma_{\mathrm{SFR}}$ in bars and bulges are in the range of $0.01-1$, one magnitude lower than the SFR ratios. In addition, the SFR densities in disks are in general 0.01 times those in bulges. The enhanced star formation in bulges indicates the effect of bar-driven internal evolution, which can make the morphological properties vary with star formation activity in galaxies.

Combining the above evolution scenarios and observational results, we derive a possible criterion to quantify the different stages of an evolutionary sequence in Figure 9. In this figure, we use the concentration index of the global SFR in the galaxy, $\mathrm{C}_{42, \mathrm{SFR}}$, and the ratio of $\Sigma_{\mathrm{SFR}}$ in the bulge and in the bar, $\frac{\Sigma_{\text {SFR bulge }}}{\Sigma_{\text {SFR bar }}}$, as the estimated parameters, and divide the parameter space into six fields (from $a$ to $f$ ) as follows.

$$
a: \frac{\Sigma_{\mathrm{SFR}_{\text {bulge }}}}{\Sigma_{\mathrm{SFR}_{\text {bar }}}}<1, C_{42, \mathrm{SFR}}<4 .
$$

This field is likely the early stage of bar-driven gas flow where a considerable fraction of the star formation activity is located in galactic outer disks, and bars have more active star formation than bulges. This stage is earlier than Type I non-starbursts in Jogee et al. (2005) because most gas is still in the outer disks.

$$
b: 1 \leqslant \frac{\Sigma_{\mathrm{SFR}_{\text {bulge }}}}{\Sigma_{\mathrm{SFR}_{\text {bar }}}}<10, C_{42, \mathrm{SFR}}<4 .
$$

This is the second stage where the gas density in bulges is higher than that in bars along with the gas inflow. Galaxies begin to have higher active star formation densities in bulges than those in bars, while the ratio is not larger than 10 , and $C_{42, \mathrm{SFR}}$ is still lower than 4 , indicating a non-negligible fraction of gas content is still in the disks.

$$
\begin{gathered}
c: 10 \leqslant \frac{\sum_{\mathrm{SFR}_{\text {bulge }}}}{\sum_{\mathrm{SFR}_{\text {bar }}}}<10^{2-C_{42, \mathrm{SFR} / 8}} \\
d: \frac{\Sigma_{\mathrm{SFR}_{\text {bulge }}}}{\sum_{\mathrm{SFR}_{\text {bar }}}} \geqslant 10^{2-C_{42, \mathrm{SFR} / 8}} .
\end{gathered}
$$

After most molecular gas has flowed into the galactic central regions, there are stages $c$ and $d$. Field $c$ is earlier in the process, corresponding to the later stage of Type II nonstarbursts in Jogee et al. (2005), where galaxies either have a low SFR concentration $C_{42, \text { SFR }}$ or have a ratio of SFR densities that is not high enough. Then as the intense star formation activity take place in bulges, galaxies in $d$ may have most of their star formation activity concentrated in their central regions and may also consume most of gas there, similar to the stage of circurmnuclear starbursts in Jogee et al. (2005).

$$
e: 1 \leqslant \frac{\Sigma_{\mathrm{SFR}_{\text {bulge }}}}{\sum_{\mathrm{SFR}_{\text {bar }}}}<10, C_{42, \mathrm{SFR}} \geqslant 4
$$




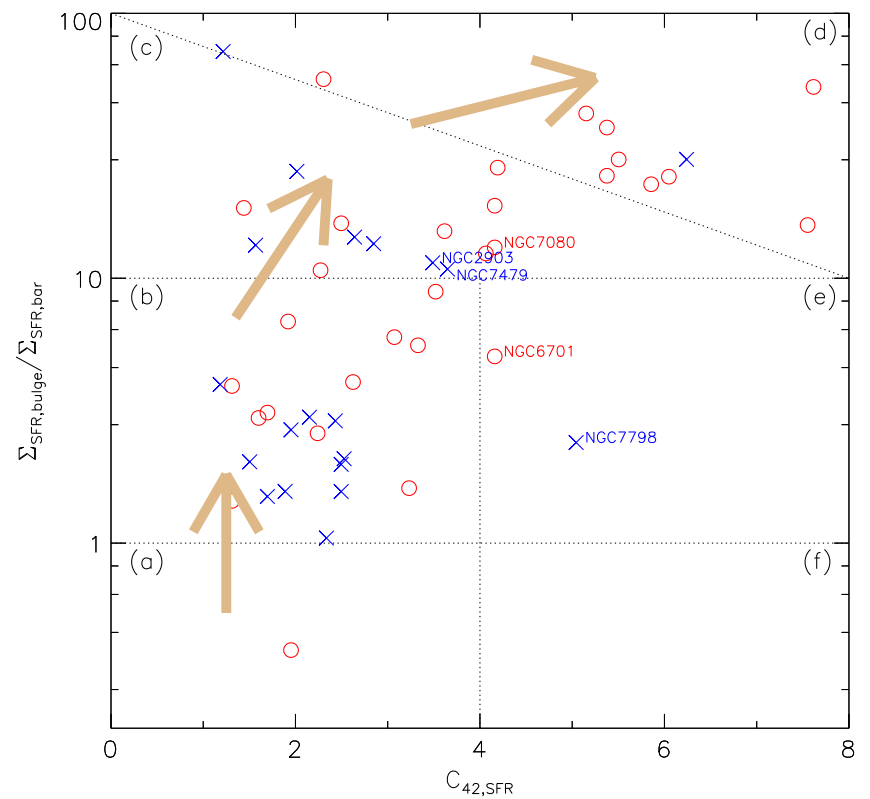

Figure 9. Comparison of the ratio of bulge-to-bar $\Sigma_{\mathrm{SFR}}$ with the concentration index of the SFR image. This plot is used as a criterion to quantify the different stages of a bar-driven evolutionary sequence (marked with arrows from $a$ to $d$ ). See the text for details. The arrows show a possible sequence of evolution. Five galaxies are specially marked in the plot and they are mentioned in the text or studied in our previous works (Zhou et al. 2011, 2012). The symbols are the same as in Figure 2.

$$
f: \frac{\Sigma_{\mathrm{SFR}_{\text {bulge }}}}{\Sigma_{\mathrm{SFR}_{\text {bar }}}}<1, C_{42, \mathrm{SFR}} \geqslant 4
$$

$e$ and $f$ have $\frac{\Sigma_{\text {SFR bulge }}}{\Sigma_{\text {SFR bar }}}$ lower than 10 but $\mathrm{C}_{42, \text { SFR }}$ larger than 4 . There are very few galaxies located in the two fields. Given our assumption, galaxies with low $\frac{\Sigma_{\text {SFR bulge }}}{\Sigma_{\text {SFR bar }}}$ are probably in the stages before circumnuclear starbursts, where galaxies should have a low SFR concentration. However, if there are other factors at play (such as galaxy interactions), which can promote the process of bar-driven gas inflow, a large fraction of molecular gas can be concentrated in the bars and bulges of galaxies even when they are still in the early stage of a bar-driven process. For example, NGC 6701 (in Field $e$ ) is apparent because it is isolated, but it has been proven to produce interactions based on its morphological and kinematical features (Marquez et al. 1996). Similarly, NGC 7798 in Field $e$ may also be in galaxy pairs and is affected by interactions (Chengalur et al. 1993). The resulting stages for our sample are listed in Table 4.

It is noted that our scenario just presents a rough criterion with uncertainties. For example, NGC 7479 is located in Stage $c$ in this scenario, where it is suggested to be in the Type I nonstarburst evolutionary phase under the effect of a minor merger (Paper I). As discussed in Section 5.1, bar-driven secular evolution is a complex process and many factors may contribute to it. To resolve this issue, more detailed observations and analysis of barred spirals are needed, along with statistical studies of large samples (e.g., using SDSS barred galaxies; F. Wang et al. 2014, in preparation).

\section{SUMMARY}

We present a study of bar-driven secular evolution from a sample of 50 nearby barred galaxies with weak and strong stellar bars. In this study, we characterize the bars using image decomposition, and estimate bar strength using the ellipticitybased parameter $f_{\text {bar }}$. Based on multi-wavelength photometry, we derive the properties of star formation activity in different stellar structures, and explore the correlations between these properties and stellar bars. The main results are as follows.

1. We use the image-fitting code BUDDA to perform a $2 \mathrm{D}$ decomposition of $3.6 \mu \mathrm{m}$ images in the bar/bulge/disk components, and obtain the structural parameters of stellar bars. Most bars in our sample are detected within less than $9 \mathrm{kpc}$, and the bars in early-type spirals are in general longer in physical and normalized lengths and stronger in strength than those in late-type ones.

2. We calculate the rotational asymmetry A and concentration index $\mathrm{C}_{42}$ to quantify the distributions of star formation activity and stellar mass in galaxies. These parameters are compared with bar strength, but no clear trends are found. We find that early-type galaxies have a higher concentration index and lower asymmetry in both SFR and stellar mass distribution than late-type ones. Weak bars tend to be associated with low concentrical star formation activity, while strong bars appear to have a large scatter in the distribution of star formation activity.

3. We explore the correlations between bar strength and SFRs, $\Sigma_{\mathrm{SFR}}$, and SSFRs in bulges and bars, as well as the global galaxies, while only weak positive trends are found between star formation activity in bulges and the bar strength along with large scatter. In addition, the early- and late-type galaxies have discernible differences in their SFRs and SSFRs, but have similar $\Sigma_{\mathrm{SFR}}$.

4. Compared with previous studies, we suggest that the differing galaxy types, stellar mass distribution, and episodes of bar-driven gas inflow may contribute to the complexity of bar-driven secular evolution, which can be used to interpret why there are no visual trends between the parameters of star formation and bar strength.

5. We find evident correlations between the star formation activity in different galactic structures, i.e., barred galaxies with intense star formation in bars tend to have active star formation in their bulges and disks, indicating a bar-driven process in galaxies.

Finally, to quantify the different stages of the process, we derived a possible criterion using the ratio of $\Sigma_{\mathrm{SFR}}$ in the bulge and the bar, and the concentration index of the galactic global SFR as the estimated parameters. While uncertainties exist because of the contribution of other factors beside stellar bars, future work is needed to explore this issue.

We are grateful to the anonymous referee for thoughtful comments and insightful suggestions that helped to improve this paper. We thank Yi-Nan Zhu for his generous help and assistance throughout the data observation and M. I. Lam for advice and helpful discussions. This project is supported by Chinese National Natural Science Foundation grants No.11303038, 11433005, 11173030, 11225316, 10978014; and also by the Strategic Priority Research Program "The Emergence of Cosmological Structures" of the Chinese Academy of Sciences, Grant No. XDB09000000. We thank 
the kind staff at the Xinglong $2.16 \mathrm{~m}$ telescope for their support during the observations. This work was partially supported by the Open Project Program of the Key Laboratory of Optical Astronomy, National Astronomical Observatories, Chinese Academy of Sciences. This work is based on observations made with the Spitzer Space Telescope, which is operated by the Jet Propulsion Laboratory, California Institute of Technology, under NASA contract 1407. We made extensive use of the NASA/IPAC Extragalactic Database (NED) which is operated by the Jet Propulsion Laboratory, California Institute of Technology, under contract with the National Aeronautics and Space Administration.

\section{REFERENCES}

Abraham, R. G., \& Merrifield, M. R. 2000, AJ, 120, 2385

Abraham, R. G., van den Bergh, S., \& Nair, P. 2003, ApJ, 588, 218

Aguerri, J. A. L., Méndez-Abreu, J., \& Corsini, E. M. 2009, A\&A, 495, 491

Alonso-Herrero, A., \& Knapen, J. H. 2001, AJ, 122, 1350

Athanassoula, E. 1992, MNRAS, 259, 345

Athanassoula, E. 2003, MNRAS, 341, 1179

Athanassoula, E., Gadotti, D. A., Carrasco, L., et al. 2009, RMxAA, 37, 79

Bershady, M. A., Jangren, A., \& Conselice, C. J. 2000, AJ, 119, 2645

Bournaud, F., \& Combes, F. 2002, A\&A, 392, 83

Bundy, K., Ellis, R. S., Conselice, C. J., et al. 2006, ApJ, 651, 120

Buta, R., \& Block, D. L. 2001, ApJ, 550, 243

Buta, R., Laurikainen, E., \& Salo, H. 2004, AJ, 127, 279

Calzetti, D., Kennicutt, R. C., Engelbracht, C. W., et al. 2007, ApJ, 666, 870

Chengalur, J. N., Salpeter, E. E., \& Terzian, Y. 1993, ApJ, 419, 30

Cheung, E., Athanassoula, E., Masters, K. L., et al. 2013, ApJ, 779, 162

Coelho, P., \& Gadotti, D. A. 2011, ApJL, 743, L13

Combes, F. 2009, in ASP Conf. Ser. 419 Galaxy Evolution: Emerging Insights and Future Challenges, ed. S. Jogee, et al. (San Francisco, CA: ASP), 31

Combes, F. 2000, in ASP Conf. Ser. 197 Dynamics of Galaxies: from the Early universe to the Present, ed. G. A. Mamon, \& V. Charmandaris (San Francisco, CA: ASP), 15

Conselice, C. J., Bershady, M. A., \& Jangren, A. 2000, ApJ, 529, 886

Cowie, L. L., Songaila, A., Hu, E. M., \& Cohen, J. G. 1996, AJ, 112, 839

Dale, D. A., Cohen, S. A., Johnson, L. C., et al. 2009, ApJ, 703, 517

Das, M., Teuben, P. J., Vogel, S. N., et al. 2003, ApJ, 582, 190

Debattista, V. P., Mayer, L., Carollo, C. M., et al. 2006, ApJ, 645, 209

de Souza, R. E., Gadotti, D. A., \& dos Anjos, S. 2004, ApJS, 153, 411

de Vaucouleurs, G., de Vaucouleurs, A., Corwin, H. G., Jr, et al. 1991, in Third

Reference Catalogue of Bright Galaxies RC3.9 C (New York: Springer)

Ellison, S. L., Nair, P., Patton, D. R., et al. 2011, MNRAS, 416, 2182

Erwin, P. 2005, MNRAS, 364, 283

Eskridge, P. B., Frogel, J. A., Pogge, R. W., et al. 2000, AJ, 119, 536

Fazio, G. G., Hora, J. L., Allen, L. E., et al. 2004, ApJS, 154, 10

Fisher, D. B. 2006, ApJ, 642, 17

Gadotti, D. A. 2008, MNRAS, 384, 420

Gadotti, D. A. 2011, MNRAS, 415, 3308

Gil de Paz, A., Boissier, S., Madore, B. F., et al. 2007, ApJS, 173, 185

Giordano, L., Tran, K. H., Moore, B., \& Saintonge, A. 2011, arXiv: 1111.1532

Hao, C.-N., Kennicutt, R. C., Johnson, B. D., et al. 2011, ApJ, 741, 124

Hancock, M., Smith, B. J., Struck, C., et al. 2007, AJ, 133, 676

Hawarden, T. G., Mountain, C. M., Leggett, S. K., \& Puxley, P. J. 1986, MNRAS, 221, 41

Helou, G., Roussel, H., Appleton, P., et al. 2004, ApJS, 154, 253

Ho, L. C., Filippenko, A. V., \& Sargent, W. L. W. 1997, ApJ, 487, 591

Hoopes, C. G., Walterbos, R. A. M., \& Bothun, G. D. 2001, ApJ, 559, 878

Huang, J. H., Gu, Q. S., Su, H. J., et al. 1996, A\&A, 313, 13

Isobe, T., \& Feigelson, E. D. 1992, ApJS, 79, 197

James, P. A., Shane, N. S., Beckman, J. E., et al. 2004, A\&A, 414, 23

Jogee, S., Barazza, F. D., Rix, H., et al. 2004, ApJ, 615, 105

Jogee, S., Scoville, N., \& Kenney, J. D. P. 2005, ApJ, 630, 837
Kent, S. M. 1985, ApJS, 59, 115

Kennicutt, R. C. 1998, ARA\&A, 36, 189

Kennicutt, R. C., Jr., Lee, J. C., Funes, S. J., et al. 2008, ApJS, 178, 247

Kennicutt, R. C., Hao, C.-N., Calzetti, D., et al. 2009, ApJ, 703, 1672

Kennicutt, R. C., Armus, L., Bendo, G., et al. 2003, PASP, 115, 928

Kim, T., Gadotti, D. A., Sheth, K., et al. 2014, ApJ, 782, 64

Knapen, J. H., Stedman, S., Bramich, D. M., Folkes, S. L., \& Bradley, T. R. 2004, A\&A, 426, 1135

Koopmann, R. A., Kenney, J. D. P., \& Young, J. 2001, ApJS, 135, 125

Kormendy, J., \& Fisher, D. B. 2005, RMxAA, 23, 101

Kormendy, J., \& Kennicutt, R. C. 2004, ARA\&A, 42, 603

Landolt, A. U. 1992, AJ, 104, 340

Laurikainen, E., Salo, H., \& Buta, R. 2004, ApJ, 607, 103

Laurikainen, E., Salo, H., Buta, R., \& Knapen, J. H. 2007, MNRAS, 381, 401

Laurikainen, E., Salo, H., \& Rautiainen, P. 2002, MNRAS, 331, 880

Lee, J. C., et al. 2008, in ASP Conf. Ser. 396, Formation and Evolution of Galaxy Disks, ed. J. G., et al. (San Francisco, CA: ASP) 109

Li, H.-N., Wu, H., Cao, C., \& Zhu, Y.-N. 2007, AJ, 134, 1315

Marquez, I., Moles, M., \& Masegosa, J. 1996, A\&A, 310, 401

Martin, P. 1995, AJ, 109, 2428

Martin, P., \& Friedli, D. 1997, A\&A, 326, 449

Meidt, S. E., Schinnerer, E., Knapen, J. H., et al. 2012, ApJ, 744, 17

Melvin, T., Masters, K., Lintott, C., et al. 2014, MNRAS, 438, 2882

Menéndez-Delmestre, K., Sheth, K., Schinnerer, E., Jarrett, et al. 2007, ApJ, 657,790

Moustakas, J., \& Kennicutt, R. C. 2006, ApJS, 164, 81

Muñoz-Mateos, J. C., Gil de Paz, A., Zamorano, J., et al. 2009, ApJ, 703, 1569

Oh, S., Oh, K., \& Yi, S. K. 2012, ApJS, 198, 4

Pahre, M. A., Ashby, M. L. N., Fazio, G. G., \& Willner, S. P. 2004a, ApJS, 154, 229

Pahre, M. A., Ashby, M. L. N., Fazio, G. G., \& Willner, S. P. 2004b, ApJS, 154,235

Piner, B. G., Stone, J. M., \& Teuben, P. J. 1995, ApJ, 449, 508

Pompea, S. M., \& Rieke, G. H. 1990, ApJ, 356, 416

Regan, M. W., \& Teuben, P. J. 2004, ApJ, 600, 595

Regan, M. W., Sheth, K., \& Vogel, S. N. 1999, ApJ, 526, 97

Regan, M. W., Vogel, S. N., \& Teuben, P. J. 1995, ApJ, 449, 576

Rieke, G. H., Young, E. T., Engelbracht, C. W., et al. 2004, ApJS, 154, 25

Roussel, H., Sauvage, M., Vigroux, L., et al. 2001, A\&A, 372, 406

Sakamoto, K., Okumura, S. K., Ishizuki, S., \& Scoville, N. Z. 1999, ApJS, 124, 403

Salpeter, E. E. 1955, ApJ, 121, 161

Sanders, D. B., Mazzarella, J. M., Kim, D.-C., Surace, J. A., \& Soifer, B. T 2003, ApJ, 126, 1607

Sellwood, J. A., \& Wilkinson, A. 1993, RPPh, 56, 173

Sérsic, J. L., \& Pastoriza, M. 1965, PASP, 77, 287

Sheth, K., Regan, M. W., Vogel, S. N., \& Teuben, P. J. 2000, ApJ, 532, 221

Sheth, K., Vogel, S. N., Regan, M. W., et al. 2002, AJ, 124, 2581

Sheth, K., Vogel, S. N., Regan, M. W., Thornley, M. D., \& Teuben, P. J. 2005 ApJ, 632, 217

Sheth, K., Elmegreen, D. M., Elmegreen, B. G., et al. 2008, ApJ, 675, 1141

Sheth, K., Regan, M. W., Hinz, J., et al. 2010, PASP, 122, 1397

Smith, B. J., Struck, C., Hancock, M., et al. 2007, AJ, 133, 791

Verley, S., Combes, F., Verdes-Montenegro, L., Bergond, G., \& Leon, S. 2007, A\&A, 474, 43

Wang, J.-L. 2008, ChJAA, 8, 643

Wang, J., Kauffmann, G., Overzier, R., et al. 2012, MNRAS, 423, 3486

Werner, M. W., Roellig, T. L., Low, F. J., et al. 2004, ApJS, 154, 1

Whyte, L. F., Abraham, R. G., Merrifield, M. R., et al. 2002, MNRAS, 336,128

Wu, H., Cao, C., Hao, C.-N., et al. 2005, ApJ, 632, 79

Wu, H., Zhu, Y. N., Cao, C., \& Qin, B. 2007, ApJ, 668, 87

Zhou, Z.-M., Cao, C., Meng, X.-M., \& Wu, H. 2011, AJ, 142, 38 (Paper I)

Zhou, Z.-M., Cao, C., \& Wu, H. 2012, RAA, 12, 235 (Paper II)

Zhu, Y.-N., Wu, H., Cao, C., \& Li, H.-N. 2008, ApJ, 686, 155

Zhu, Y.-N., Wu, H., Li, H.-N., \& Cao, C. 2010, RAA, 10, 329

Zurita, A., \& Pérez, I. 2008, A\&A, 485, 5 